\title{
Copper-Catalyzed Divergent Addition Reactions of Enoldiazoacetamides with Nitrones
}

\author{
Qing-Qing Cheng, Julietta Yedoyan, Hadi Arman, and Michael P. Doyle* \\ Department of Chemistry, The University of Texas at San Antonio, San Antonio, \\ Texas 78249, United States
}

\section{Table of Contents}

1. General Information

2. Copper-Catalyzed Addition Reactions of Enoldiazo Compounds 1c-1e with Nitrone 2a.

3. General Procedure for Copper-Catalyzed [3 + 3]-Cycloaddition and Analytical Data for the

Products.

4. General Procedure for Copper-Catalyzed Mannich Addition and Analytical Data for the

Products.

5. Experimental Studies of Mechanism.

6. X-Ray Diffraction Analysis for Compound 3ab

7. References

8. NMR Spectra for Compounds 3 and $\mathbf{4}$.

9. HPLC Analyses for Compounds 3 


\section{General Information}

All reactions, unless noted, were performed in oven-dried $\left(120^{\circ} \mathrm{C}\right)$ glassware with magnetic stirring under an inert atmosphere of dry nitrogen. Analytical thin layer chromatography (TLC) was carried out using Dynamic Adsorbents precoated $(0.25 \mathrm{~mm}$ thickness $)$ silica gel plates with $\mathrm{F}_{254}$ indicator. Visualization was accomplished by UV light $(254 \mathrm{~nm})$ or phosphomolybdic acid (PMA) solution in ethanol. Liquid chromatography was performed using a forced flow (flash chromatography) of the indicated system on silica gel (230-400 mesh). Melting points were obtained uncorrected from an Electro Thermo Mel-Temp DLX 104 device. Proton nuclear magnetic resonance $\left({ }^{1} \mathrm{H}\right.$ NMR) spectra were recorded in $\mathrm{CDCl}_{3}$ on an Agilent DD2-500 spectrometer (500 MHz). Chemical shifts were reported as $\delta$ in ppm downfield from internal $\mathrm{Me}_{4} \mathrm{Si}$. Multiplicities were given as: s (singlet); $\mathrm{d}$ (doublet); t (triplet); q (quartet); dd (doublet of doublets); qd (quartet of doublets); m (multiplet); comp (composite). The number of protons $(n)$ for a given resonance was indicated by $n \mathrm{H}$. Coupling constants were reported as $J$ values in $\mathrm{Hz}$. Carbon nuclear magnetic resonance $\left({ }^{13} \mathrm{C}\right.$ NMR) spectra were recorded in $\mathrm{CDCl}_{3}$ on an Agilent DD2-500 spectrometer $(126 \mathrm{MHz})$ with complete proton decoupling. Enantiomeric excesses were determined on an Agilent 1200 Series HPLC using Daicel Chiralpak AD-H or Chiralcel OD-H columns. High-resolution mass spectra (HRMS) were obtained on a Bruker micrOTOF mass spectrometer with ESI resource using CsI as standard.

Metal salts and ligands were purchased from Aldrich or Strem and used without further purification. Enoldiazo compounds $\mathbf{1}^{1}$, nitrones $\mathbf{2}^{2}$, and cyclopropene $\mathbf{7} \mathbf{a}^{1 \mathrm{a}}$ were prepared according to the literature procedures. Solvents were dried according to standard procedures. ${ }^{3}$ All the other chemicals were obtained from commercial sources and used as received. 


\section{Copper-Catalyzed Addition Reactions of Enoldiazo Compounds 1c-1e with Nitrone 2a}

Enoldiazoacetamides bearing methyl (1c) and ethyl (1d) substituents at the vinylogous position were investigated (Table S1). Both copper(I) tetrafluoroborate (entries 2 and 3) and copper(I) triflate (entries 8 and 9) gave the annulation products (3ca/3da) in excellent yields (90-96\%) with exceptional diastereocontrol ( $\mathrm{dr}>20: 1)$, and moderate to good enantioselectivities were obtained in the presence of chiral ligand 6 (entries 5 and 6). Plausible reasons for the different product selectivities (entries 8 and 9 $v s$. entry 7) are as follows: (i) copper(I) triflate prefers to facilitate dinitrogen extrusion from enoldiazoacetamides $\mathbf{1 c}$ and $\mathbf{1 d}$ to trigger the [3+3]-cycloaddition process, whereas enoldiazoacetamide 1a is relatively inert in the presence of copper(I) triflate (see Scheme 3a in the Manuscript); (ii) methyl (1c) and ethyl (1d) substituents at the vinylogous position of enoldiazoacetamides are unfavorable for vinylogous association with the more sterically hindered electrophilic site of nitrones, which could lead to the Mannich addition pathway.

Table S1. Copper-Catalyzed Addition Reactions of Enoldiazoacetamides 1 with Nitrone 2a ${ }^{a}$

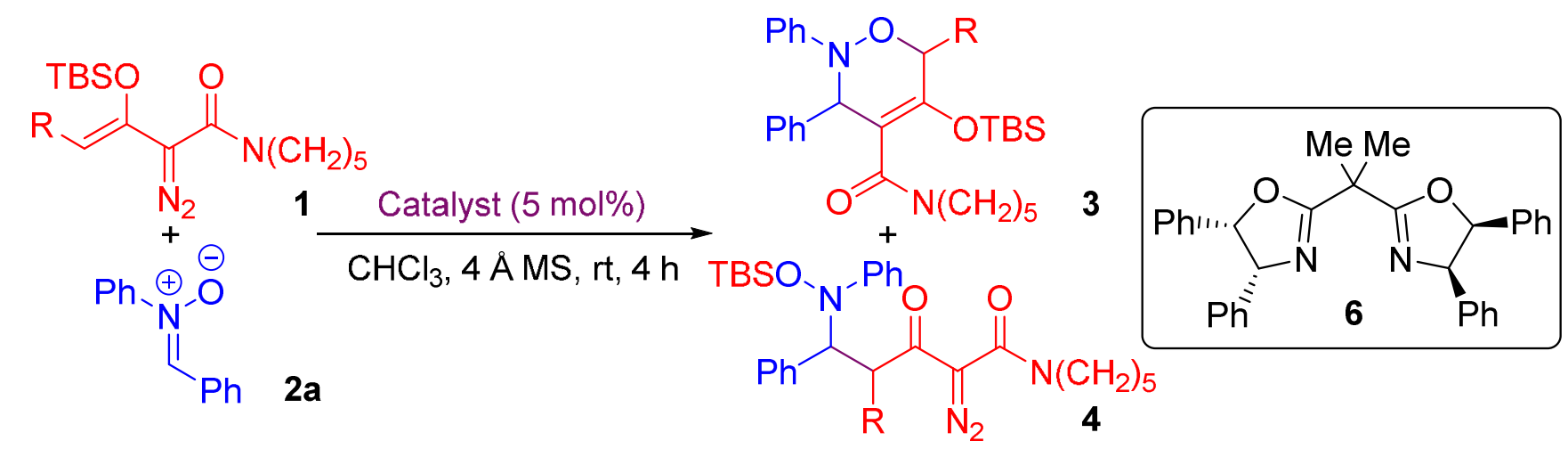

\begin{tabular}{cccccccc}
\hline entry & $\mathbf{1}$ & $\mathrm{R}$ & catalyst & yield of $\mathbf{3}(\%)^{b}$ & $\mathrm{dr}$ of $\mathbf{3}^{c}$ & ee of $\mathbf{3}(\%)^{d}$ & yield of $\mathbf{4}(\%)^{b, e}$ \\
\hline 1 & $\mathbf{1 a}$ & $\mathrm{H}$ & $\mathrm{Cu}(\mathrm{MeCN})_{4} \mathrm{BF}_{4}$ & 61 & -- & -- & n.d. \\
2 & $\mathbf{1 c}$ & $\mathrm{Me}$ & $\mathrm{Cu}(\mathrm{MeCN})_{4} \mathrm{BF}_{4}$ & 96 & $>20: 1$ & -- & n.d. \\
3 & $\mathbf{1 d}$ & $\mathrm{Et}$ & $\mathrm{Cu}(\mathrm{MeCN})_{4} \mathrm{BF}_{4}$ & 96 & $>20: 1$ & -- & n.d. \\
$4^{f}$ & $\mathbf{1 a}$ & $\mathrm{H}$ & $\mathrm{Cu}(\mathrm{MeCN})_{4} \mathrm{BF}_{4} / \mathbf{6}$ & 96 & -- & 98 & n.d. \\
$5^{f}$ & $\mathbf{1 c}$ & $\mathrm{Me}$ & $\mathrm{Cu}(\mathrm{MeCN})_{4} \mathrm{BF}_{4} / \mathbf{6}$ & 90 & $>20: 1$ & 86 & n.d. \\
$6^{f}$ & $\mathbf{1 d}$ & $\mathrm{Et}$ & $\mathrm{Cu}(\mathrm{MeCN})_{4} \mathrm{BF}_{4} / \mathbf{6}$ & 94 & $>20: 1$ & 67 & n.d. \\
\hline
\end{tabular}




\begin{tabular}{|c|c|c|c|c|c|c|c|}
\hline 7 & $1 \mathbf{a}$ & $\mathrm{H}$ & $\mathrm{CuOTf} \cdot \mathrm{Tol}_{1 / 2}$ & trace & -- & -- & 88 \\
\hline 8 & 1c & $\mathrm{Me}$ & $\mathrm{CuOTf} \cdot \mathrm{Tol}_{1 / 2}$ & 90 & $>20: 1$ & -- & n.d. \\
\hline 9 & 1d & $\mathrm{Et}$ & $\mathrm{CuOTf} \cdot \mathrm{Tol}_{1 / 2}$ & 96 & $>20: 1$ & -- & n.d. \\
\hline
\end{tabular}

${ }^{a}$ Reaction conditions: catalyst:1:2a $=0.01: 0.24: 0.2(\mathrm{mmol})$, with $4 \AA$ molecular sieves $(50 \mathrm{mg})$ in chloroform $(2 \mathrm{~mL})$ at room temperature for $4 \mathrm{~h} .{ }^{b}$ Isolated yields after flash column chromatography. ${ }^{c}$ Diastereomeric ratios were determined by ${ }^{1} \mathrm{H}$ NMR analysis of the reaction mixtures. ${ }^{d}$ Enantiomeric excesses were determined by chiral HPLC analysis. ${ }^{e}$ n.d. $=$ not detectable by ${ }^{1} \mathrm{H}$ NMR and TLC of the reaction mixture. ${ }^{f}$ The catalyst was generated in situ from $\mathrm{Cu}(\mathrm{MeCN})_{4} \mathrm{BF}_{4}(0.01 \mathrm{mmol})$ and chiral ligand $6(0.012 \mathrm{mmol})$.

Methyl enoldiazoacetate (1e) was also examined in the present catalytic system (Table S2), and the reactions with enoldiazoacetate $1 \mathrm{e}$ showed the same product selectivity as those with enoldiazoacetamide 1a (Table S1, entries 4 and 7). Either [3 +3]-cycloaddition or Mannich addition was achieved in high yield with absolute product exclusivity by the use of corresponding copper catalyst.

Table S2. Copper-Catalyzed Addition Reactions of Enoldiazoacetate 1e with Nitrone $\mathbf{2 a}^{a}$

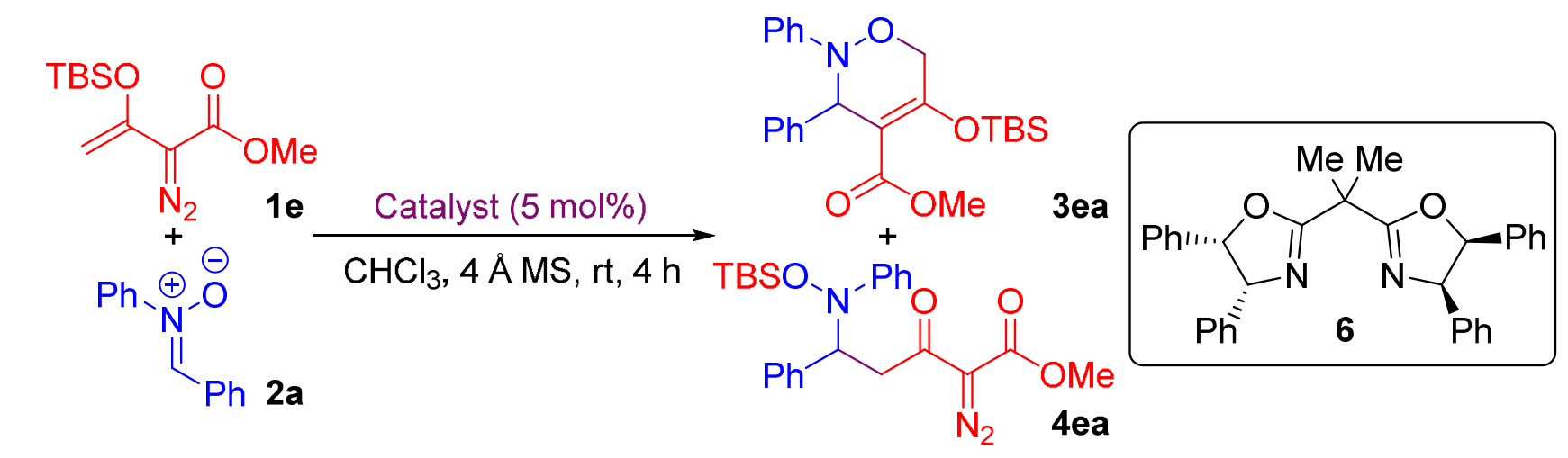

\begin{tabular}{ccccc}
\hline entry & catalyst & yield of $\mathbf{3 e a}(\%)^{b, c}$ & ee of $\mathbf{3 e a}(\%)^{d}$ & yield of $\mathbf{4 e a}(\%)^{b, c}$ \\
\hline $1^{e}$ & $\mathrm{Cu}(\mathrm{MeCN})_{4} \mathrm{BF}_{4} / \mathbf{6}$ & 87 & 39 & n.d. \\
2 & $\mathrm{CuOTf} \cdot \mathrm{Tol}_{1 / 2}$ & n.d. & -- & 94 \\
\hline
\end{tabular}

${ }^{a}$ Reaction conditions: catalyst:1e:2a $=0.01: 0.24: 0.2(\mathrm{mmol})$, with $4 \AA$ molecular sieves $(50 \mathrm{mg})$ in chloroform $(2 \mathrm{~mL})$ at room temperature for $4 \mathrm{~h} .{ }^{b}$ Isolated yields after flash column chromatography. ${ }^{c}$ n.d. = not detectable by ${ }^{1} \mathrm{H}$ NMR and TLC of the reaction mixture. ${ }^{d}$ Enantiomeric excesse was determined by chiral HPLC analysis. ${ }^{e}$ The catalyst was generated in situ from $\mathrm{Cu}(\mathrm{MeCN})_{4} \mathrm{BF}_{4}(0.01$ $\mathrm{mmol})$ and chiral ligand 6 (0.012 $\mathrm{mmol})$. 


\section{General Procedure for Copper-Catalyzed [3 + 3]-Cycloaddition and Analytical Data for the}

\section{Products}

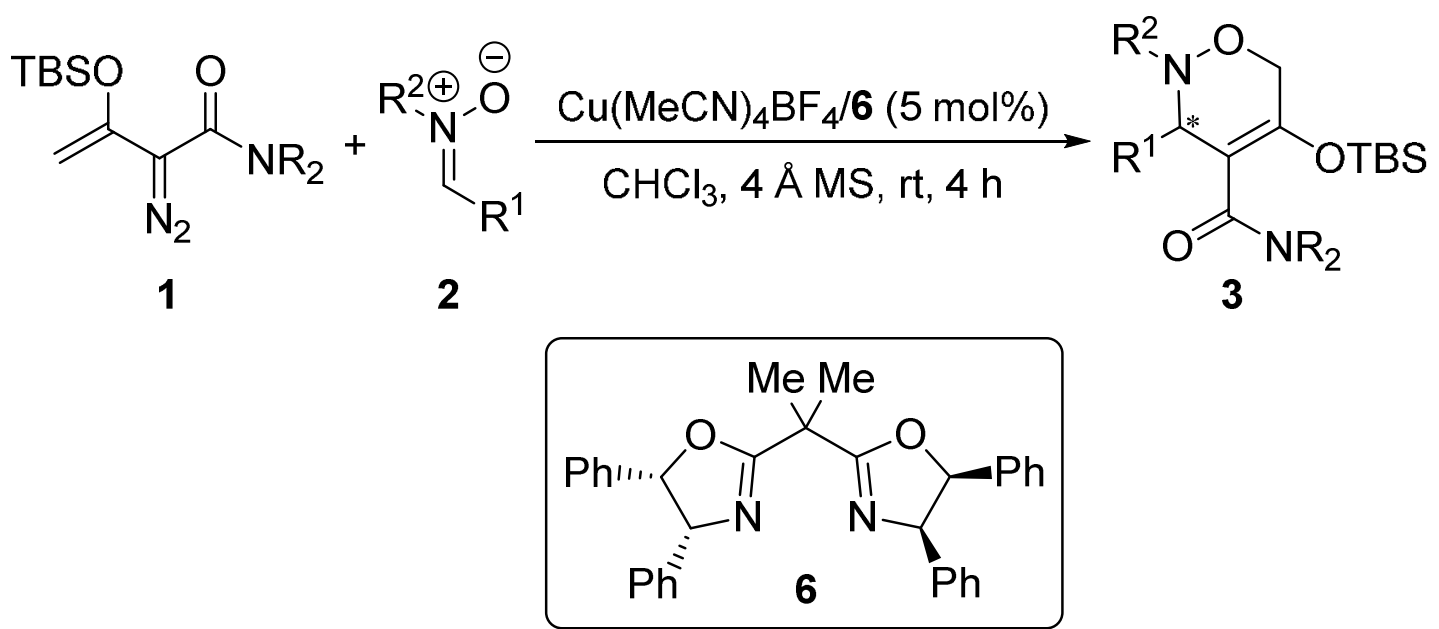

$\mathrm{Cu}(\mathrm{MeCN})_{4} \mathrm{BF}_{4}(3.1 \mathrm{mg}, 0.01 \mathrm{mmol}, 5 \mathrm{~mol} \%)$, ligand $6(5.8 \mathrm{mg}, 0.012 \mathrm{mmol}, 6 \mathrm{~mol} \%), 4 \AA$ molecular sieves $(50 \mathrm{mg}$, activated), and dry chloroform $(2 \mathrm{~mL})$ were sequentially introduced into an oven-dried Schlenk tube under a flow of dry nitrogen. The resulting mixture was stirred at room temperature under the nitrogen atmosphere for $2 \mathrm{~h}$. Then nitrone $2(0.2 \mathrm{mmol})$ and enoldiazoacetamide 1 $(0.24 \mathrm{mmol})$ were sequentially introduced into the system, and stirring was continued at room temperature for $4 \mathrm{~h}$. Subsequently, the reaction mixture was concentrated in vacuo, and the residue was purified by flash chromatography on silica gel using hexanes/ethyl acetate as eluent to afford the corresponding [3 +3]-cycloaddition product 3. Analytical data for these products were listed below.

\section{[5-(tert-Butyldimethylsilyloxy)-2,3-diphenyl-3,6-dihydro-2H-1,2-oxazin-4-yl](piperidin-1-yl)metha-} none (3aa)<smiles>CN(C)C(=O)C1=C([OH2+])CON(c2ccccc2)C1c1ccccc1</smiles>
White solid, m.p. $140-141{ }^{\circ} \mathrm{C} .96 \%$ yield, $98 \%$ ee [HPLC conditions: Chiralpak AD-H column, hexanes $/{ }^{i} \mathrm{PrOH}=80: 20$, flow rate $=1.0 \mathrm{~mL} / \mathrm{min}$, wavelength $=$ $254 \mathrm{~nm}, t_{\mathrm{R}}=6.06 \mathrm{~min}$ for major isomer, $t_{\mathrm{R}}=15.14 \mathrm{~min}$ for minor isomer]. ${ }^{1} \mathrm{H}$ NMR (500 MHz, $\left.\mathrm{CDCl}_{3}\right) \delta 7.41(\mathrm{~d}, J=7.2 \mathrm{~Hz}, 2 \mathrm{H}), 7.23-7.03$ (comp, 7H), 6.85 (t, $J=7.2 \mathrm{~Hz}, 1 \mathrm{H}), 5.64(\mathrm{~s}, 1 \mathrm{H}), 4.36(\mathrm{~d}, J=14.9 \mathrm{~Hz}, 1 \mathrm{H}), 4.25(\mathrm{~d}, J=13.3 \mathrm{~Hz}$, 1H), $4.20(\mathrm{~d}, J=14.9 \mathrm{~Hz}, 1 \mathrm{H}), 3.49$ (d, $J=13.3 \mathrm{~Hz}, 1 \mathrm{H}), 2.97$ (t, $J=11.8 \mathrm{~Hz}, 1 \mathrm{H}), 2.68$ (t, $J=11.8 \mathrm{~Hz}$, 1H), 1.51-1.45 (comp, 2H), 1.33-1.22 (comp, 2H), 1.18-1.10 (m, 1H), $0.89(\mathrm{~s}, 9 \mathrm{H}), 0.30-0.22(\mathrm{~m}, 1 \mathrm{H})$, 0.08 (s, 3H), 0.07 (s, 3H). ${ }^{13} \mathrm{C}$ NMR $\left(126 \mathrm{MHz}, \mathrm{CDCl}_{3}\right) \delta 165.72,147.58,144.16,138.52,128.95$, $128.59,128.01,127.31,121.73,116.67,113.70,67.45,62.24,47.49,42.48,25.88,25.43,25.36,24.43$, 
17.90, -4.29, -4.42. HRMS (ESI) calcd for $\left[\mathrm{C}_{28} \mathrm{H}_{38} \mathrm{~N}_{2} \mathrm{O}_{3} \mathrm{Si}+\mathrm{Na}, \mathrm{M}+\mathrm{Na}\right]^{+}:$501.2544, Found: 501.2546.

\section{(S)-[5-(tert-Butyldimethylsilyloxy)-3-(4-chlorophenyl)-2-phenyl-3,6-dihydro-2H-1,2-oxazin-4-yl]} (piperidin-1-yl)methanone (3ab)<smiles>CN(C)C(=O)C1=C([OH2+])CON(c2ccccc2)[C@H]1c1ccc(Cl)cc1</smiles>

White solid, m.p. $151-152{ }^{\circ} \mathrm{C} .94 \%$ yield, $98 \%$ ee [HPLC conditions: Chiralpak AD-H column, hexanes $/ \mathrm{PrOH}=80: 20$, flow rate $=1.0$ $\mathrm{mL} /$ min, wavelength $=254 \mathrm{~nm}, t_{\mathrm{R}}=8.67$ min for $(S)$-enantiomer, $t_{\mathrm{R}}=$ $11.59 \mathrm{~min}$ for $(R)$-enantiomer]. ${ }^{1} \mathrm{H}$ NMR $\left(500 \mathrm{MHz}, \mathrm{CDCl}_{3}\right) \delta 7.34(\mathrm{~d}, J$ $=7.5 \mathrm{~Hz}, 2 \mathrm{H}), 7.26-7.10(\mathrm{comp}, 4 \mathrm{H}), 7.06(\mathrm{~d}, J=8.0 \mathrm{~Hz}, 2 \mathrm{H}), 6.87(\mathrm{t}, J$

$=7.5 \mathrm{~Hz}, 1 \mathrm{H}), 5.63(\mathrm{~s}, 1 \mathrm{H}), 4.36(\mathrm{~d}, J=14.9 \mathrm{~Hz}, 1 \mathrm{H}), 4.23(\mathrm{~d}, J=13.4 \mathrm{~Hz}, 1 \mathrm{H}), 4.19(\mathrm{~d}, J=14.9 \mathrm{~Hz}$, 1H), 3.47 (d, $J=13.4 \mathrm{~Hz}, 1 \mathrm{H}), 3.00$ (t, $J=11.6 \mathrm{~Hz}, 1 \mathrm{H}), 2.72$ (t, $J=11.6 \mathrm{~Hz}, 1 \mathrm{H}), 1.58-1.48$ (comp, 2H), 1.39-1.26 (comp, 2H), 1.21-1.11 (m, 1H), 0.89 (s, 9H), 0.42-0.31 (m, 1H), 0.08 (s, 3H), 0.07 (s, $3 \mathrm{H}) .{ }^{13} \mathrm{C}$ NMR $\left(126 \mathrm{MHz}, \mathrm{CDCl}_{3}\right) \delta 165.55,147.37,144.83,137.10,133.18,130.34,128.68,128.13$, $122.00,116.64,113.30,67.61,61.82,47.54,42.56,26.13,25.43,24.41,17.92,-4.25,-4.41$. HRMS (ESI) calcd for $\left[\mathrm{C}_{28} \mathrm{H}_{37} \mathrm{ClN}_{2} \mathrm{O}_{3} \mathrm{Si}+\mathrm{Na}, \mathrm{M}+\mathrm{Na}\right]^{+}:$535.2154, Found: 535.2151 .

\section{[5-(tert-Butyldimethylsilyloxy)-2-phenyl-3-(p-tolyl)-3,6-dihydro-2H-1,2-oxazin-4-yl](piperidin-1-yl)} methanone (3ac)<smiles>Cc1ccc(C2C(C(=O)N(C)C)=C([OH2+])CON2c2ccccc2)cc1</smiles>
White solid, m.p. $140-141{ }^{\circ} \mathrm{C} .96 \%$ yield, $96 \%$ ee [HPLC conditions: Chiralpak AD-H column, hexanes $/{ }^{i} \mathrm{PrOH}=90: 10$, flow rate $=1.0$ $\mathrm{mL} / \mathrm{min}$, wavelength $=254 \mathrm{~nm}, t_{\mathrm{R}}=11.87$ min for major isomer, $t_{\mathrm{R}}=$ $16.32 \mathrm{~min}$ for minor isomer]. ${ }^{1} \mathrm{H} \mathrm{NMR}\left(500 \mathrm{MHz}, \mathrm{CDCl}_{3}\right) \delta$ 7.31-7.26 (comp, 2H), 7.21-7.15 (comp, 2H), 7.10 (d, $J=8.0 \mathrm{~Hz}, 2 \mathrm{H}), 6.97$ (d, $J$ $=7.5 \mathrm{~Hz}, 2 \mathrm{H}), 6.84(\mathrm{t}, J=7.5 \mathrm{~Hz}, 1 \mathrm{H}), 5.61(\mathrm{~s}, 1 \mathrm{H}), 4.35(\mathrm{~d}, J=14.9 \mathrm{~Hz}, 1 \mathrm{H}), 4.23(\mathrm{~d}, J=13.3 \mathrm{~Hz}, 1 \mathrm{H})$, 4.19 (d, $J=14.9 \mathrm{~Hz}, 1 \mathrm{H}), 3.49$ (d, $J=13.3 \mathrm{~Hz}, 1 \mathrm{H}), 2.99$ (t, $J=11.4 \mathrm{~Hz}, 1 \mathrm{H}), 2.71(\mathrm{t}, J=11.4 \mathrm{~Hz}, 1 \mathrm{H})$, 2.22 (s, 3H), 1.52-1.45 (comp, 2H), 1.35-1.24 (comp, 2H), 1.20-1.12 (m, 1H), 0.89 (s, 9H), 0.38-0.29 (m, 1H), 0.07 (s, 3H), 0.06 (s, 3H). ${ }^{13} \mathrm{C}$ NMR (126 MHz, $\left.\mathrm{CDCl}_{3}\right) \delta$ 165.77, 147.61, 144.00, 136.76, 135.31, 128.81, 128.61, 128.53, 121.60, 116.63, 113.77, 67.37, 61.91, 47.48, 42.43, 25.85, 25.41, 25.33, 24.44, 21.03, 17.86, -4.31, -4.47. HRMS (ESI) calcd for $\left[\mathrm{C}_{29} \mathrm{H}_{40} \mathrm{~N}_{2} \mathrm{O}_{3} \mathrm{Si}+\mathrm{Na}, \mathrm{M}+\mathrm{Na}\right]^{+}:$515.2700, Found: 515.2697.

[5-(tert-Butyldimethylsilyloxy)-3-(4-methoxyphenyl)-2-phenyl-3,6-dihydro-2H-1,2-oxazin-4-yl](piperidin-1-yl)methanone (3ad) 


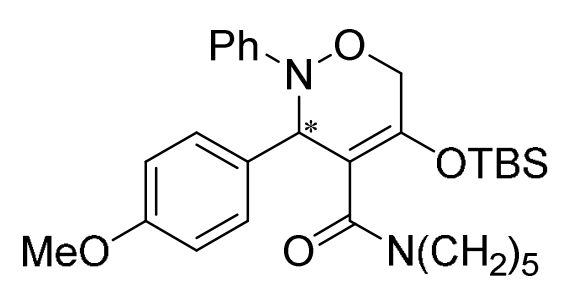

White solid, m.p. $119-120{ }^{\circ} \mathrm{C} .95 \%$ yield, $96 \%$ ee [HPLC conditions: Chiralpak AD-H column, hexanes $/ \mathrm{PrOH}=80: 20$, flow rate $=1.0$ $\mathrm{mL} / \mathrm{min}$, wavelength $=230 \mathrm{~nm}, t_{\mathrm{R}}=8.58$ min for major isomer, $t_{\mathrm{R}}=$ $19.69 \mathrm{~min}$ for minor isomer]. ${ }^{1} \mathrm{H}$ NMR $\left(500 \mathrm{MHz}, \mathrm{CDCl}_{3}\right) \delta 7.31(\mathrm{~d}, J$ $=7.5 \mathrm{~Hz}, 2 \mathrm{H}), 7.21-7.15(\mathrm{comp}, 2 \mathrm{H}), 7.08(\mathrm{~d}, J=8.0 \mathrm{~Hz}, 2 \mathrm{H}), 6.85(\mathrm{t}$, $J=7.5 \mathrm{~Hz}, 1 \mathrm{H}), 6.74-6.67$ (comp, 2H), 5.59 (s, 1H), 4.36 (d, $J=14.9 \mathrm{~Hz}, 1 \mathrm{H}), 4.22(\mathrm{~d}, J=13.4 \mathrm{~Hz}, 1 \mathrm{H})$, 4.19 (d, $J=14.9 \mathrm{~Hz}, 1 \mathrm{H}), 3.71$ (s, 3H), 3.51 (d, $J=13.4 \mathrm{~Hz}, 1 \mathrm{H}), 3.01$ (t, $J=11.2 \mathrm{~Hz}, 1 \mathrm{H}), 2.72$ (t, $J=$ $11.2 \mathrm{~Hz}, 1 \mathrm{H}), 1.54-1.46$ (comp, 2H), 1.36-1.27 (comp, 2H), 1.20-1.12 (m, 1H), 0.89 (s, 9H), 0.45-0.36 (m, 1H), 0.08 (s, 3H), 0.07 (s, 3H). ${ }^{13} \mathrm{C}$ NMR $\left(126 \mathrm{MHz}, \mathrm{CDCl}_{3}\right) \delta 165.78,158.78,147.64,144.08$, $130.49,130.15,128.53,121.69,116.74,113.83,113.32,67.50,61.86,55.09,47.52,42.46,26.03,25.43$, 25.37, 24.44, 17.88, -4.28, -4.45. HRMS (ESI) calcd for $\left[\mathrm{C}_{29} \mathrm{H}_{40} \mathrm{~N}_{2} \mathrm{O}_{4} \mathrm{Si}+\mathrm{Na}, \mathrm{M}+\mathrm{Na}\right]^{+}:$531.2650, Found: 531.2655 .

[5-(tert-Butyldimethylsilyloxy)-3-(3-chlorophenyl)-2-phenyl-3,6-dihydro-2H-1,2-oxazin-4-yl](piperidin-1-yl)methanone (3ae)<smiles>CCN(C)C(=O)C1=C(O[Sb])CON(c2ccccc2)C1c1cccc(Cl)c1</smiles>
White solid, m.p. $116-117^{\circ} \mathrm{C} .90 \%$ yield, $96 \%$ ee [HPLC conditions: Chiralpak AD-H column, hexanes $/ \mathrm{PrOH}=80: 20$, flow rate $=1.0$ $\mathrm{mL} / \mathrm{min}$, wavelength $=230 \mathrm{~nm}, t_{\mathrm{R}}=5.58$ min for major isomer, $t_{\mathrm{R}}=$ $14.50 \mathrm{~min}$ for minor isomer]. ${ }^{1} \mathrm{H} \mathrm{NMR}\left(500 \mathrm{MHz}, \mathrm{CDCl}_{3}\right) \delta 7.46(\mathrm{~s}, 1 \mathrm{H})$, 7.27-7.24 (m, 1H), 7.23-7.17 (comp, 2H), 7.16-7.04 (comp, 4H), 6.88 (t, $J=7.3 \mathrm{~Hz}, 1 \mathrm{H}), 5.64$ (s, 1H), 4.34 (d, $J=15.0 \mathrm{~Hz}, 1 \mathrm{H}), 4.31$ (d, $J=13.2 \mathrm{~Hz}, 1 \mathrm{H}), 4.19$ (d, $J=15.0$ $\mathrm{Hz}, 1 \mathrm{H}), 3.52(\mathrm{~d}, J=13.2 \mathrm{~Hz}, 1 \mathrm{H}), 2.98(\mathrm{t}, J=11.5 \mathrm{~Hz}, 1 \mathrm{H}), 2.67$ (t, $J=11.5 \mathrm{~Hz}, 1 \mathrm{H}), 1.57-1.49$ (comp, 2H), 1.39-1.29 (comp, 2H), 1.21-1.13 (m, 1H), 0.88 (s, 9H), 0.39-0.30 (m, 1H), 0.07 (s, 3H), 0.05 (s, $3 \mathrm{H}) .{ }^{13} \mathrm{C}$ NMR $\left(126 \mathrm{MHz}, \mathrm{CDCl}_{3}\right) \delta 165.44,147.21,144.81,140.75,133.90,129.25,128.78,128.69$, 127.48, 127.17, 122.04, 116.69, 113.07, 67.26, 61.65, 47.56, 42.55, 25.96, 25.43, 25.39, 24.41, 17.87, $-4.33,-4.46$. HRMS (ESI) calcd for $\left[\mathrm{C}_{28} \mathrm{H}_{37} \mathrm{ClN}_{2} \mathrm{O}_{3} \mathrm{Si}+\mathrm{Na}, \mathrm{M}+\mathrm{Na}\right]^{+}:$535.2154, Found: 535.2158.

[5-(tert-Butyldimethylsilyloxy)-3-(naphthalen-2-yl)-2-phenyl-3,6-dihydro-2H-1,2-oxazin-4-yl](piperidin-1-yl)methanone (3af)<smiles>CN(C)C(=O)C1=C(O[Sb])CON(c2ccccc2)C1c1ccc2ccccc2c1</smiles>

White solid, m.p. $147-148{ }^{\circ} \mathrm{C} .94 \%$ yield, $97 \%$ ee [HPLC conditions: Chiralpak AD-H column, hexanes $/ \mathrm{PrOH}=80: 20$, flow rate $=1.0$ $\mathrm{mL} / \mathrm{min}$, wavelength $=230 \mathrm{~nm}, t_{\mathrm{R}}=9.16$ min for major isomer, $t_{\mathrm{R}}=$ $20.81 \mathrm{~min}$ for minor isomer]. ${ }^{1} \mathrm{H}$ NMR $\left(500 \mathrm{MHz}, \mathrm{CDCl}_{3}\right) \delta 7.82(\mathrm{~s}$, 1H), 7.76-7.70 (comp, 2H), 7.69-7.62 (comp, 2H), 7.43-7.36 (comp, 
2H), 7.22-7.11 (comp, 4H), 6.86-6.81 (m, 1H), $5.86(\mathrm{~s}, 1 \mathrm{H}), 4.42$ (d, J=14.9 Hz, 1H), $4.27(\mathrm{~d}, J=14.9$ $\mathrm{Hz}, 1 \mathrm{H}), 4.22$ (d, $J=13.2 \mathrm{~Hz}, 1 \mathrm{H}), 3.49$ (d, $J=13.2 \mathrm{~Hz}, 1 \mathrm{H}), 2.98$ (t, $J=11.2 \mathrm{~Hz}, 1 \mathrm{H}), 2.69$ (t, $J=11.2$ $\mathrm{Hz}, 1 \mathrm{H}), 1.44-1.38$ (m, 1H), 1.28-1.12 (comp, 3H), 1.06-0.99 (m, 1H), 0.91 (s, 9H), 0.18--0.02 (comp, 7H). ${ }^{13} \mathrm{C} \mathrm{NMR}\left(126 \mathrm{MHz}, \mathrm{CDCl}_{3}\right) \delta 165.72,147.49,144.44,136.12,133.03,132.65,128.60,127.96$, 127.86, 127.68, 127.40, 126.68, 125.71, 125.59, 121.76, 116.64, 113.56, 67.40, 62.22, 47.46, 42.43, 25.79, 25.42, 25.25, 24.23, 17.88, -4.28, -4.44. HRMS (ESI) calcd for $\left[\mathrm{C}_{32} \mathrm{H}_{40} \mathrm{~N}_{2} \mathrm{O}_{3} \mathrm{Si}+\mathrm{Na}, \mathrm{M}+\mathrm{Na}\right]^{+}$: 551.2700, Found: 551.2704.

[5-(tert-Butyldimethylsilyloxy)-3-(furan-2-yl)-2-phenyl-3,6-dihydro-2H-1,2-oxazin-4-yl](piperidin1-yl)methanone (3ag)

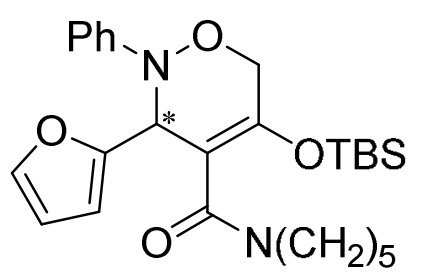

White solid, m.p. $125-126{ }^{\circ} \mathrm{C}$. 91\% yield, 98\% ee [HPLC conditions: Chiralpak AD-H column, hexanes $/{ }^{i} \mathrm{PrOH}=90: 10$, flow rate $=1.0 \mathrm{~mL} / \mathrm{min}$, wavelength $=220 \mathrm{~nm}, t_{\mathrm{R}}=10.55 \mathrm{~min}$ for major isomer, $t_{\mathrm{R}}=12.60 \min$ for minor isomer]. ${ }^{1} \mathrm{H}$ NMR $\left(500 \mathrm{MHz}, \mathrm{CDCl}_{3}\right) \delta 7.31-7.21$ (comp, 3H), 7.16 (d, $J=7.8 \mathrm{~Hz}, 2 \mathrm{H}), 6.98-6.92(\mathrm{~m}, 1 \mathrm{H}), 6.20(\mathrm{dd}, J=3.1,1.8 \mathrm{~Hz}, 1 \mathrm{H}), 6.14(\mathrm{~d}, J$ $=3.1 \mathrm{~Hz}, 1 \mathrm{H}), 5.73(\mathrm{~s}, 1 \mathrm{H}), 4.34(\mathrm{dd}, J=15.0,1.8 \mathrm{~Hz}, 1 \mathrm{H}), 4.29(\mathrm{~d}, J=12.9 \mathrm{~Hz}, 1 \mathrm{H}), 4.17(\mathrm{~d}, J=15.0$ $\mathrm{Hz}, 1 \mathrm{H}), 3.78(\mathrm{~d}, J=12.9 \mathrm{~Hz}, 1 \mathrm{H}), 3.15$ (t, $J=11.3 \mathrm{~Hz}, 1 \mathrm{H}), 2.80(\mathrm{t}, J=11.3 \mathrm{~Hz}, 1 \mathrm{H}), 1.68-1.62(\mathrm{~m}$, 1H), 1.60-1.42 (comp, 3H), 1.37-1.29 (m, 1H), 1.04-0.80 (comp, 10H), 0.08 (s, 3H), $0.05(\mathrm{~s}, 3 \mathrm{H}) .{ }^{13} \mathrm{C}$ NMR $\left(126 \mathrm{MHz}, \mathrm{CDCl}_{3}\right) \delta 165.52,151.25,147.20,145.66,142.03,128.60,122.44,117.01,110.99$, 110.06, 109.22, 67.14, 56.71, 47.68, 42.57, 26.32, 25.44, 25.40, 24.54, 17.88, -4.26, -4.50. HRMS (ESI) calcd for $\left[\mathrm{C}_{26} \mathrm{H}_{36} \mathrm{~N}_{2} \mathrm{O}_{4} \mathrm{Si}+\mathrm{Na}, \mathrm{M}+\mathrm{Na}\right]^{+}$: 491.2337, Found: 491.2340.

\section{Ethyl 4-[5-(tert-Butyldimethylsilyloxy)-3-phenyl-4-(piperidine-1-carbonyl)-3,6-dihydro-2H-1,2- oxazin-2-yl]benzoate (3ah)}

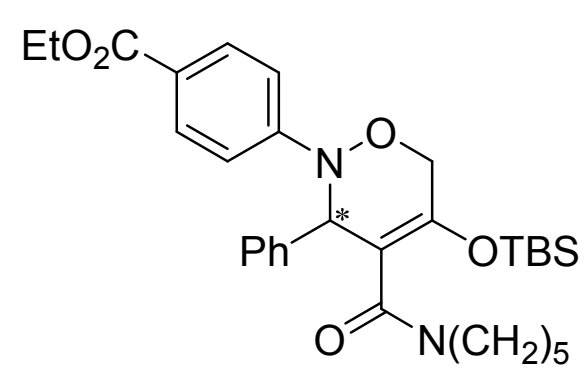

White solid, m.p. $152-153{ }^{\circ} \mathrm{C} . \quad 92 \%$ yield, 93\% ee [HPLC conditions: Chiralcel OD-H column, hexanes $/{ }^{i} \mathrm{PrOH}=98: 2$, flow rate $=1.0 \mathrm{~mL} / \mathrm{min}$, wavelength $=254 \mathrm{~nm}, t_{\mathrm{R}}=8.49 \mathrm{~min}$ for major isomer, $t_{\mathrm{R}}=9.80 \mathrm{~min}$ for minor isomer]. ${ }^{1} \mathrm{H} \mathrm{NMR}(500 \mathrm{MHz}$, $\left.\mathrm{CDCl}_{3}\right) \delta 7.89-7.81$ (comp, 2H), $7.40(\mathrm{~d}, J=7.3 \mathrm{~Hz}, 2 \mathrm{H}), 7.20-7.08$ (comp, 3H), 7.07-6.98 (comp, 2H), 5.73 (s, 1H), 4.48 (d, $J=14.8$ $\mathrm{Hz}, 1 \mathrm{H}), 4.34-4.20$ (comp, 4H), 3.42 (d, $J=13.2 \mathrm{~Hz}, 1 \mathrm{H}), 2.93$ (t, $J=11.8 \mathrm{~Hz}, 1 \mathrm{H}), 2.67$ (t, $J=11.8 \mathrm{~Hz}$ 1H), 1.50-1.43 (comp, 2H), 1.34-1.10 (comp, 6H), 0.90 (s, 9H), 0.23-0.04 (comp, 7H). ${ }^{13} \mathrm{C}$ NMR (126 
$\left.\mathrm{MHz}, \mathrm{CDCl}_{3}\right) \delta 166.46,165.36,151.18,143.88,137.96,130.54,128.62,128.20,127.53,122.53,114.32$, $113.74,68.71,61.66,60.42,47.47,42.52,25.80,25.41,25.35,24.37,17.90,14.34,-4.27,-4.34$. HRMS (ESI) calcd for $\left[\mathrm{C}_{31} \mathrm{H}_{42} \mathrm{~N}_{2} \mathrm{O}_{5} \mathrm{Si}+\mathrm{Na}, \mathrm{M}+\mathrm{Na}\right]^{+}:$573.2755, Found: 573.2759.

\section{[2-(3-Bromo-4-methylphenyl)-5-(tert-butyldimethylsilyloxy)-3-phenyl-3,6-dihydro-2H-1,2-oxazin-}

\section{4-yl](piperidin-1-yl)methanone (3ai)}

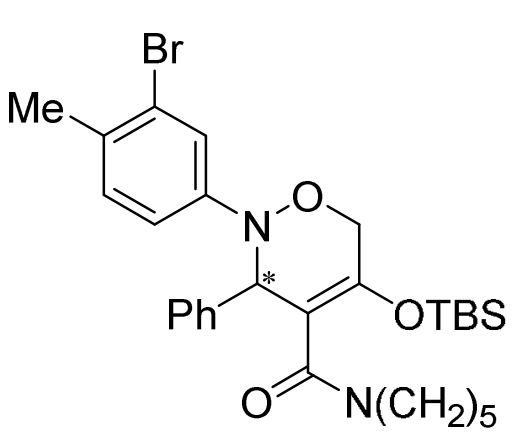

White solid, m.p. $146-147{ }^{\circ} \mathrm{C} .96 \%$ yield, 94\% ee [HPLC conditions: Chiralpak AD-H column, hexanes $/{ }^{i} \mathrm{PrOH}=80: 20$, flow rate $=1.0$ $\mathrm{mL} / \mathrm{min}$, wavelength $=210 \mathrm{~nm}, t_{\mathrm{R}}=5.89 \min$ for major isomer, $t_{\mathrm{R}}=$ $10.38 \mathrm{~min}$ for minor isomer]. ${ }^{1} \mathrm{H} \mathrm{NMR}\left(500 \mathrm{MHz}, \mathrm{CDCl}_{3}\right) \delta 7.39(\mathrm{~d}, J$ $=7.5 \mathrm{~Hz}, 2 \mathrm{H}), 7.34(\mathrm{~s}, 1 \mathrm{H}), 7.20-7.11(\mathrm{comp}, 3 \mathrm{H}), 6.98(\mathrm{~d}, J=8.3 \mathrm{~Hz}$, $1 \mathrm{H}), 6.83(\mathrm{~d}, J=8.3 \mathrm{~Hz}, 1 \mathrm{H}), 5.55(\mathrm{~s}, 1 \mathrm{H}), 4.38(\mathrm{~d}, J=14.8 \mathrm{~Hz}, 1 \mathrm{H})$, $4.24(\mathrm{~d}, J=13.4 \mathrm{~Hz}, 1 \mathrm{H}), 4.21(\mathrm{~d}, J=14.8 \mathrm{~Hz}, 1 \mathrm{H}), 3.44(\mathrm{~d}, J=13.4$ $\mathrm{Hz}, 1 \mathrm{H}), 2.94(\mathrm{t}, J=12.0 \mathrm{~Hz}, 1 \mathrm{H}), 2.66(\mathrm{t}, J=12.0 \mathrm{~Hz}, 1 \mathrm{H}), 2.22$ (s, 3H), 1.50-1.43 (comp, 2H), 1.32-1.20 (comp, 2H), 1.14-1.07 (m, 1H), 0.89 (s, 9H), 0.23-0.16 (m, 1H), 0.10 (s, 3H), 0.09 (s, 3H). ${ }^{13} \mathrm{C}$ NMR $\left(126 \mathrm{MHz}, \mathrm{CDCl}_{3}\right) \delta 165.50,146.60,143.98,138.07,130.41,130.37,128.89,128.07,127.42$, $124.81,120.35,115.20,113.71,67.99,62.41,47.44,42.45,25.82,25.40,25.32,24.37,21.88,17.87$, $-4.29,-4.39$. HRMS (ESI) calcd for $\left[\mathrm{C}_{29} \mathrm{H}_{39} \mathrm{BrN}_{2} \mathrm{O}_{3} \mathrm{Si}+\mathrm{Na}, \mathrm{M}+\mathrm{Na}\right]^{+}$: 593.1806, Found: 593.1803.

\section{[2-Benzyl-5-(tert-butyldimethylsilyloxy)-3-cyclohexyl-3,6-dihydro-2H-1,2-oxazin-4-yl](piperidin-1-} yl)methanone (3aj)

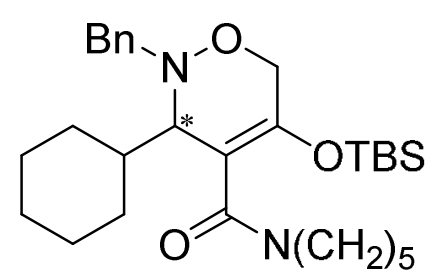

Colorless oil. 93\% yield, 94\% ee [HPLC conditions: Chiralpak AD-H column, hexanes $/{ }^{i} \mathrm{PrOH}=95: 5$, flow rate $=1.0 \mathrm{~mL} / \mathrm{min}$, wavelength $=230 \mathrm{~nm}, t_{\mathrm{R}}=$ $7.97 \mathrm{~min}$ for major isomer, $t_{\mathrm{R}}=9.07 \mathrm{~min}$ for minor isomer]. ${ }^{1} \mathrm{H}$ NMR (500 $\left.\mathrm{MHz} \mathrm{CDCl}_{3}\right) \delta 7.44(\mathrm{~d}, J=7.3 \mathrm{~Hz}, 2 \mathrm{H}), 7.37-7.27$ (comp, 2H), 7.24 (t, $J=$ $7.3 \mathrm{~Hz}, 1 \mathrm{H}), 4.36(\mathrm{~d}, J=13.5 \mathrm{~Hz}, 1 \mathrm{H}), 4.06(\mathrm{~d}, J=12.6 \mathrm{~Hz}, 1 \mathrm{H}), 4.02$ (dd, $J$ $=16.0,2.0 \mathrm{~Hz}, 1 \mathrm{H}), 3.85(\mathrm{~d}, J=12.6 \mathrm{~Hz}, 1 \mathrm{H}), 3.83(\mathrm{~d}, J=13.5 \mathrm{~Hz}, 1 \mathrm{H}), 3.75(\mathrm{~d}, J=16.0 \mathrm{~Hz}, 1 \mathrm{H}), 3.51$ (s, 1H), 3.24-3.16 (m, 1H), 2.88-2.80 (m, 1H), 1.80-1.08 (comp, 17H), 0.93 (s, 9H), 0.21 (s, 3H), 0.16 (s, 3H). ${ }^{13} \mathrm{C}$ NMR $\left(126 \mathrm{MHz}, \mathrm{CDCl}_{3}\right) \delta 167.13,142.66,136.90,129.28,128.22,127.27,110.45,63.74$, 59.42, 57.45, 47.62, 42.49, 42.34, 30.83, 29.09, 27.00, 26.55, 26.42, 26.39, 25.50, 24.65, 17.95, -3.93, -4.29. HRMS (ESI) calcd for $\left[\mathrm{C}_{29} \mathrm{H}_{46} \mathrm{~N}_{2} \mathrm{O}_{3} \mathrm{Si}+\mathrm{Na}, \mathrm{M}+\mathrm{Na}\right]^{+}: 521.3170$, Found: 521.3172 . 


\section{5-(tert-Butyldimethylsilyloxy)- $N, N$-dimethyl-2,3-diphenyl-3,6-dihydro-2H-1,2-oxazine-4-carboxa-}

mide (3ba)<smiles>CN(C)C(=O)C1=C([O+]S(C)(=O)=O)CON(c2ccccc2)C1c1ccccc1</smiles>

White solid, m.p. $132-133{ }^{\circ}$ C. $96 \%$ yield, 96\% ee [HPLC conditions: Chiralpak AD-H column, hexanes $/{ }^{i} \mathrm{PrOH}=80: 20$, flow rate $=1.0 \mathrm{~mL} / \mathrm{min}$, wavelength $=$ $210 \mathrm{~nm}, t_{\mathrm{R}}=6.28 \mathrm{~min}$ for major isomer, $t_{\mathrm{R}}=14.63 \mathrm{~min}$ for minor isomer]. ${ }^{1} \mathrm{H}$ NMR (500 MHz, $\left.\mathrm{CDCl}_{3}\right) \delta 7.36$ (d, $\left.J=7.4 \mathrm{~Hz}, 2 \mathrm{H}\right), 7.24-7.13$ (comp, 5H), 7.11 $(\mathrm{d}, J=8.1 \mathrm{~Hz}, 2 \mathrm{H}), 6.87$ (t, $J=7.4 \mathrm{~Hz}, 1 \mathrm{H}), 5.64(\mathrm{~s}, 1 \mathrm{H}), 4.35$ (d, $J=15.0 \mathrm{~Hz}$,

$1 \mathrm{H}), 4.20(\mathrm{~d}, J=15.0 \mathrm{~Hz}, 1 \mathrm{H}), 2.84(\mathrm{~s}, 3 \mathrm{H}), 2.80(\mathrm{~s}, 3 \mathrm{H}), 0.89(\mathrm{~s}, 9 \mathrm{H}), 0.07(\mathrm{~s}, 3 \mathrm{H}), 0.05(\mathrm{~s}, 3 \mathrm{H}) .{ }^{13} \mathrm{C}$ NMR $\left(126 \mathrm{MHz}, \mathrm{CDCl}_{3}\right) \delta 167.56,147.46,145.28,138.02,128.92,128.59,127.86,127.40,122.02$, 117.06, 113.47, 67.07, 62.36, 37.51, 34.68, 25.36, 17.84, -4.39, -4.52. HRMS (ESI) calcd for $\left[\mathrm{C}_{25} \mathrm{H}_{34} \mathrm{~N}_{2} \mathrm{O}_{3} \mathrm{Si}+\mathrm{Na}, \mathrm{M}+\mathrm{Na}\right]^{+}: 461.2231$, Found: 461.2228.

[5-(tert-Butyldimethylsilyloxy)-6-methyl-2,3-diphenyl-3,6-dihydro-2H-1,2-oxazin-4-yl](piperidin-1yl)methanone (3ca)<smiles>CCN(C)C(=O)C1=C([OH2+])C(C)ON(c2ccccc2)C1c1ccccc1</smiles>

Colorless oil. 90\% yield, $\mathrm{dr}>20: 1,86 \%$ ee [HPLC conditions: Chiralpak AD-H column, hexanes $/{ }^{i} \mathrm{PrOH}=80: 20$, flow rate $=1.0 \mathrm{~mL} / \mathrm{min}$, wavelength $=254 \mathrm{~nm}$, $t_{\mathrm{R}}=6.15 \mathrm{~min}$ for major isomer, $t_{\mathrm{R}}=18.87 \mathrm{~min}$ for minor isomer]. ${ }^{1} \mathrm{H}$ NMR (500 $\left.\mathrm{MHz}_{\mathrm{CDCl}}\right) \delta 7.43(\mathrm{~d}, J=7.2 \mathrm{~Hz}, 2 \mathrm{H}), 7.26-6.99$ (comp, 7H), 6.83 (t, $J=7.2$ $\mathrm{Hz}, 1 \mathrm{H}), 5.68(\mathrm{~s}, 1 \mathrm{H}), 4.45(\mathrm{qd}, J=6.6,1.8 \mathrm{~Hz}, 1 \mathrm{H}), 4.31(\mathrm{~d}, J=12.5 \mathrm{~Hz}, 1 \mathrm{H})$, $3.53(\mathrm{~d}, J=12.5 \mathrm{~Hz}, 1 \mathrm{H}), 2.95(\mathrm{t}, J=11.3 \mathrm{~Hz}, 1 \mathrm{H}), 2.60(\mathrm{t}, J=11.3 \mathrm{~Hz}, 1 \mathrm{H}), 1.51(\mathrm{~d}, J=6.6 \mathrm{~Hz}, 3 \mathrm{H})$, 1.51-1.44 (comp, 2H), 1.30-1.24 (comp, 2H), 1.14-1.07 (m, 1H), $0.91(\mathrm{~s}, 9 \mathrm{H}), 0.29-0.21(\mathrm{~m}, 1 \mathrm{H}), 0.06$ (s, 3H), 0.05 (s, 3H). ${ }^{13} \mathrm{C}$ NMR (126 MHz, $\left.\mathrm{CDCl}_{3}\right) \delta 165.90,147.99,147.75,138.44,128.99,128.54$, $128.00,127.26,121.40,116.38,113.94,72.98,63.15,47.42,42.49$, 25.91, 25.59, 25.34, 24.44, 17.99, 16.29, -4.41, -4.48. HRMS (ESI) calcd for $\left[\mathrm{C}_{29} \mathrm{H}_{40} \mathrm{~N}_{2} \mathrm{O}_{3} \mathrm{Si}+\mathrm{Na}, \mathrm{M}+\mathrm{Na}\right]^{+}:$515.2700, Found: 515.2696.

\section{[5-(tert-Butyldimethylsilyloxy)-6-ethyl-2,3-diphenyl-3,6-dihydro-2H-1,2-oxazin-4-yl](piperidin-1-} yl)methanone (3da)<smiles>CCCCN(C)C(=O)C1=C([OH2+])C(c2ccccc2)=C(c2ccccc2)NO1</smiles>

Colorless oil. 94\% yield, dr > 20:1, 67\% ee [HPLC conditions: Chiralpak AD-H column, hexanes $/{ }^{i} \mathrm{PrOH}=90: 10$, flow rate $=1.0 \mathrm{~mL} / \mathrm{min}$, wavelength $=254 \mathrm{~nm}$, $t_{\mathrm{R}}=7.31 \mathrm{~min}$ for major isomer, $t_{\mathrm{R}}=12.91 \mathrm{~min}$ for minor isomer]. ${ }^{1} \mathrm{H} \mathrm{NMR}(500$ $\left.\mathrm{MHz} \mathrm{CDCl}_{3}\right) \delta 7.45(\mathrm{~d}, J=7.2 \mathrm{~Hz}, 2 \mathrm{H}), 7.23-7.05$ (comp, 7H), $6.81(\mathrm{t}, J=7.2$ $\mathrm{Hz}, 1 \mathrm{H}), 5.72$ (s, 1H), 4.36-4.27 (comp, 2H), 3.50 (d, J=13.4 Hz, 1H), 2.94 (t, $J$ 
$=11.2 \mathrm{~Hz}, 1 \mathrm{H}), 2.63(\mathrm{t}, J=11.2 \mathrm{~Hz}, 1 \mathrm{H}), 2.18-1.89(\mathrm{comp}, 2 \mathrm{H}), 1.51-1.45$ (comp, 2H), 1.30-1.21 (comp, 2H), $1.15(\mathrm{t}, J=7.4 \mathrm{~Hz}, 3 \mathrm{H}), 1.13-1.08(\mathrm{~m}, 1 \mathrm{H}), 0.91(\mathrm{~s}, 9 \mathrm{H}), 0.26-0.20(\mathrm{~m}, 1 \mathrm{H}), 0.07(\mathrm{~s}, 3 \mathrm{H})$, $0.06(\mathrm{~s}, 3 \mathrm{H}) .{ }^{13} \mathrm{C}$ NMR $\left(126 \mathrm{MHz}, \mathrm{CDCl}_{3}\right) \delta 166.00,147.83,147.02,138.61,128.79,128.49,127.97$, 127.11, 121.13, 116.04, 114.96, 77.80, 62.89, 47.32, 42.43, 25.76, 25.55, 25.29, 24.37, 23.54, 17.94, 9.89, -4.40, -4.51. HRMS (ESI) calcd for $\left[\mathrm{C}_{30} \mathrm{H}_{42} \mathrm{~N}_{2} \mathrm{O}_{3} \mathrm{Si}+\mathrm{Na}, \mathrm{M}+\mathrm{Na}\right]^{+}:$529.2857, Found: 529.2861. 


\section{General Procedure for Copper-Catalyzed Mannich Addition and Analytical Data for the}

\section{Products}

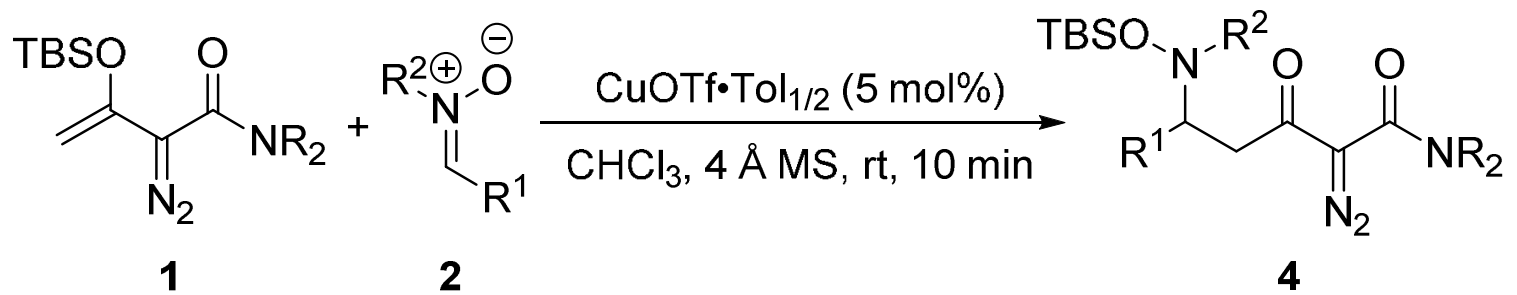

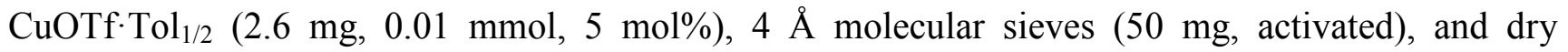
chloroform $(2 \mathrm{~mL})$ were sequentially introduced into an oven-dried Schlenk tube under a flow of dry nitrogen. The resulting mixture was stirred at room temperature under the nitrogen atmosphere for $5 \mathrm{~min}$. Then nitrone $2(0.2 \mathrm{mmol})$ and enoldiazoacetamide $\mathbf{1}(0.24 \mathrm{mmol})$ were sequentially introduced into the system, and stirring was continued at room temperature for $10 \mathrm{~min}$. Subsequently, the reaction mixture was concentrated in vacuo, and the residue was purified by flash chromatography on silica gel using hexanes/ethyl acetate as eluent to afford the corresponding Mannich addition product 4 . Analytical data for these products were listed below.

\section{5-[(tert-Butyldimethylsilyloxy)(phenyl)amino]-2-diazo-5-phenyl-1-(piperidin-1-yl)pentane-1,3-} dione (4aa)<smiles>CCCCCCN(C)C(=O)C(=[W])CC(c1ccccc1)N(P)O[AsH3]</smiles>

Light yellow oil. $88 \%$ yield. ${ }^{1} \mathrm{H}$ NMR $\left(500 \mathrm{MHz}, \mathrm{CDCl}_{3}\right) \delta$ 7.26-7.10 (comp, 7H), 7.06 (d, $J=7.5 \mathrm{~Hz}, 2 \mathrm{H}), 6.99$ (t, $J=7.5 \mathrm{~Hz}$, $1 \mathrm{H}), 4.87$ (dd, $J=8.6,5.7 \mathrm{~Hz}, 1 \mathrm{H}), 3.47-3.04$ (comp, 6H), 1.63-1.47 (comp, 6H), 0.91 (s, 9H), -0.11 (s, 3H), -0.45 (s, 3H). ${ }^{13} \mathrm{C}$ NMR (126 $\left.\mathrm{MHz}, \mathrm{CDCl}_{3}\right) \delta 159.77,152.23,137.70,129.73,128.07,127.75,127.68,123.90,120.78,70.29,70.24$, $46.49,38.99,26.16,25.75,24.33,17.99,-4.88,-5.67$. HRMS (ESI) calcd for $\left[\mathrm{C}_{28} \mathrm{H}_{38} \mathrm{~N}_{4} \mathrm{O}_{3} \mathrm{Si}+\mathrm{Na}, \mathrm{M}+\right.$ $\mathrm{Na}]^{+}:$529.2605, Found: 529.2604.

\section{5-[(tert-Butyldimethylsilyloxy)(phenyl)amino]-5-(4-chlorophenyl)-2-diazo-1-(piperidin-1-yl)pen-} tane-1,3-dione (4ab)<smiles>CCCCCN(C)C(=O)C(=[W])C(=O)CC(c1ccc(Cl)cc1)N(OS(C)(=O)=O)c1ccccc1</smiles>

Light yellow oil. $84 \%$ yield. ${ }^{1} \mathrm{H}$ NMR $\left(500 \mathrm{MHz}, \mathrm{CDCl}_{3}\right) \delta$ 7.26-7.14 (comp, 4H), 7.10 (d, $J=8.0 \mathrm{~Hz}, 2 \mathrm{H}$ ), 7.06-6.96 (comp, 3H), 4.84 (dd, $J=8.2,6.0 \mathrm{~Hz}, 1 \mathrm{H}$ ), 3.48-3.10 (comp, 6H), 1.64-1.51 (comp, 6H), 0.91 (s, 9H), -0.06 (s, 3H), -0.39 (s, $3 \mathrm{H}) .{ }^{13} \mathrm{C} \mathrm{NMR}\left(126 \mathrm{MHz}, \mathrm{CDCl}_{3}\right) \delta 159.71,152.05,136.02$, 
$133.51,131.04,128.15,127.75,124.09,120.79,69.53,69.49,46.50,39.65,26.14,25.75,24.34,17.98$, $-4.83,-5.44$. HRMS (ESI) calcd for $\left[\mathrm{C}_{28} \mathrm{H}_{37} \mathrm{ClN}_{4} \mathrm{O}_{3} \mathrm{Si}+\mathrm{Na}, \mathrm{M}+\mathrm{Na}\right]^{+}: 563.2216$, Found: 563.2211.

\section{5-[(tert-Butyldimethylsilyloxy)(phenyl)amino]-2-diazo-5-(naphthalen-2-yl)-1-(piperidin-1-yl)pen-} tane-1,3-dione (4af)<smiles>CN(C)C(=O)C(=O)CC(c1ccc2ccccc2c1)N(OC(F)(F)F)P1c2ccccc21</smiles>

Light yellow oil. $86 \%$ yield. ${ }^{1} \mathrm{H}$ NMR $\left(500 \mathrm{MHz}, \mathrm{CDCl}_{3}\right) \delta$ 7.90-7.77 (m, 1H), 7.77-7.64 (comp, 2H), $7.62(\mathrm{~s}, 1 \mathrm{H})$, 7.53-7.27 (comp, 3H), 7.18 (t, $J=7.5 \mathrm{~Hz}, 2 \mathrm{H}), 7.09$ (d, $J=7.5$ $\mathrm{Hz}, 2 \mathrm{H}), 7.02-6.93(\mathrm{~m}, 1 \mathrm{H}), 5.03(\mathrm{dd}, J=8.5,5.7 \mathrm{~Hz}, 1 \mathrm{H})$, 3.64-2.90 (comp, 6H), 1.69-1.29 (comp, 6H), 0.92 (s, 9H), -0.09 (s, 3H), $-0.50(\mathrm{~s}, 3 \mathrm{H}) .{ }^{13} \mathrm{C} \mathrm{NMR}\left(126 \mathrm{MHz}, \mathrm{CDCl}_{3}\right) \delta 159.72,152.22,135.27,132.95,132.83$, $128.58,128.12$, 128.03, 128.00, 127.46, 127.18, 125.86, 125.81, 123.97, 120.82, 70.48, 46.46, 39.29, $26.15,25.61,24.28,17.96,-4.85,-5.55$. HRMS (ESI) calcd for $\left[\mathrm{C}_{32} \mathrm{H}_{40} \mathrm{~N}_{4} \mathrm{O}_{3} \mathrm{Si}+\mathrm{Na}, \mathrm{M}+\mathrm{Na}\right]^{+}$: 579.2762, Found: 579.2767.

Ethyl 4-[(tert-Butyldimethylsilyloxy)(4-diazo-3,5-dioxo-1-phenyl-5-(piperidin-1-yl)pentyl)amino] benzoate (4ah)<smiles>CCOC(=O)c1ccc(N(C(CC(=O)C(=N)N(C)C)c2ccccc2)C(C)(C)C)cc1</smiles>
Light yellow oil. $92 \%$ yield. ${ }^{1} \mathrm{H}$ NMR $\left(500 \mathrm{MHz}, \mathrm{CDCl}_{3}\right) \delta 7.88(\mathrm{~d}, J$ $=8.5 \mathrm{~Hz}, 2 \mathrm{H}), 7.25-7.14(\mathrm{comp}, 5 \mathrm{H}), 7.10(\mathrm{~d}, J=8.5 \mathrm{~Hz}, 2 \mathrm{H}), 5.03$ (dd, $J=7.9,6.1 \mathrm{~Hz}, 1 \mathrm{H}), 4.33$ (q, $J=7.1 \mathrm{~Hz}, 2 \mathrm{H}), 3.47-3.11$ (comp, 6H), 1.64-1.49 (comp, 6H), 1.37 (t, $J=7.1 \mathrm{~Hz}, 3 \mathrm{H}), 0.94$ (s, 9H), $-0.05(\mathrm{~s}, 3 \mathrm{H}),-0.47$ (s, 3H). ${ }^{13} \mathrm{C}$ NMR $\left(126 \mathrm{MHz}, \mathrm{CDCl}_{3}\right) \delta 166.36$, $159.73,156.73,136.99,129.90,129.57,127.96,127.79,125.37$, $119.57,69.72,69.67,60.66,46.50,39.07,26.14,25.72,24.31,18.02,14.34,-4.77,-5.65$. HRMS (ESI) calcd for $\left[\mathrm{C}_{31} \mathrm{H}_{42} \mathrm{~N}_{4} \mathrm{O}_{5} \mathrm{Si}+\mathrm{Na}, \mathrm{M}+\mathrm{Na}\right]^{+}: 601.2817$, Found: 601.2816 . 


\section{Experimental Studies of Mechanism}

a)

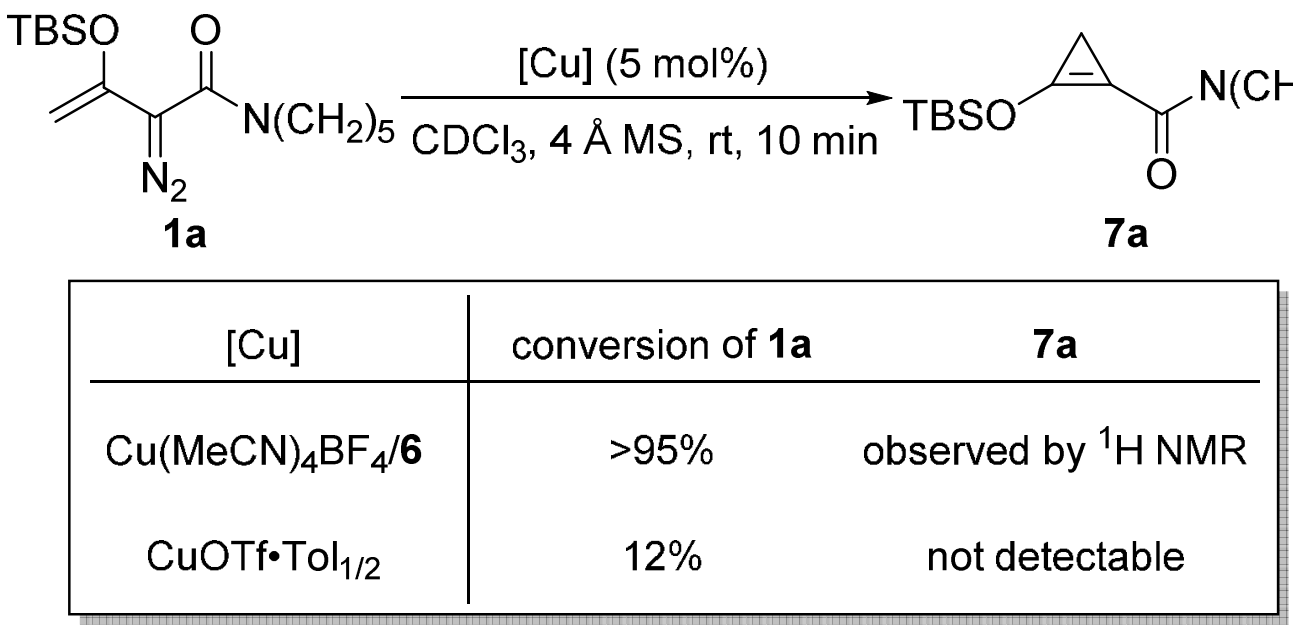

$\mathrm{Cu}(\mathrm{MeCN})_{4} \mathrm{BF}_{4}(3.1 \mathrm{mg}, 0.01 \mathrm{mmol}, 5 \mathrm{~mol} \%)$, ligand 6 (5.8 mg, $\left.0.012 \mathrm{mmol}, 6 \mathrm{~mol} \%\right), 4 \AA$ molecular sieves $\left(50 \mathrm{mg}\right.$, activated), and dry $\mathrm{CDCl}_{3}(2 \mathrm{~mL})$ were sequentially introduced into an oven-dried Schlenk tube under a flow of dry nitrogen. The resulting mixture was stirred at room temperature under the nitrogen atmosphere for $2 \mathrm{~h}$. Then enoldiazoacetamide $1 \mathrm{a}$ (74 $\mathrm{mg}, 0.24 \mathrm{mmol})$ was introduced into the system, and stirring was continued at room temperature. Part of the reaction mixure $(0.3 \mathrm{~mL})$ was transferred into an oven-dried NMR spectroscopy tube under a flow of dry nitrogen at specified times, and ${ }^{1} \mathrm{H}$ NMR spectra were recorded on an Agilent DD2-500 spectrometer (500 MHz).

$\mathrm{CuOTf} \mathrm{Tol}_{1 / 2}(2.6 \mathrm{mg}, 0.01 \mathrm{mmol}, 5 \mathrm{~mol} \%), 4 \AA$ molecular sieves (50 mg, activated), and dry $\mathrm{CDCl}_{3}$ $(2 \mathrm{~mL})$ were sequentially introduced into an oven-dried Schlenk tube under a flow of dry nitrogen. The resulting mixture was stirred at room temperature under the nitrogen atmosphere for $5 \mathrm{~min}$. Then enoldiazoacetamide 1a (74 mg, $0.24 \mathrm{mmol}$ ) was introduced into the system, and stirring was continued at room temperature. Part of the reaction mixure $(0.3 \mathrm{~mL})$ was transferred into an oven-dried NMR spectroscopy tube under a flow of dry nitrogen at specified times, and ${ }^{1} \mathrm{H}$ NMR spectra were recorded on an Agilent DD2-500 spectrometer (500 MHz). 


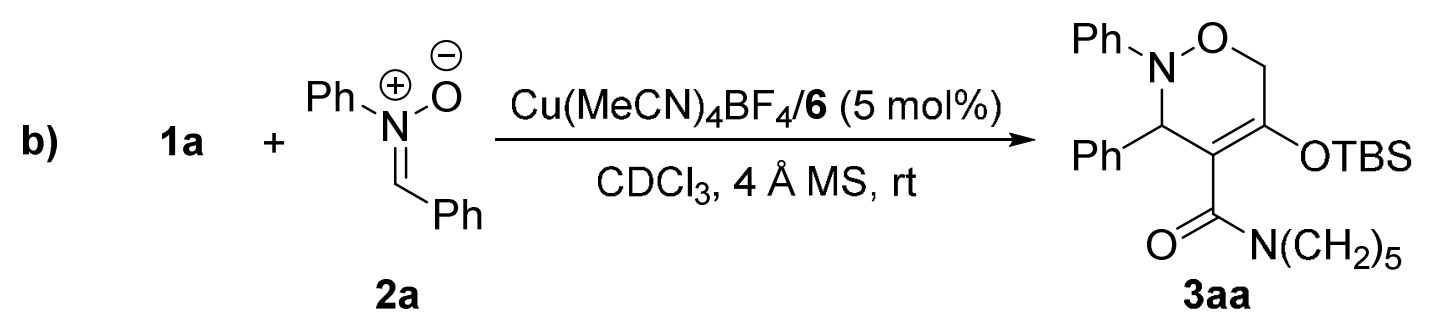

$4 \mathrm{~h}$
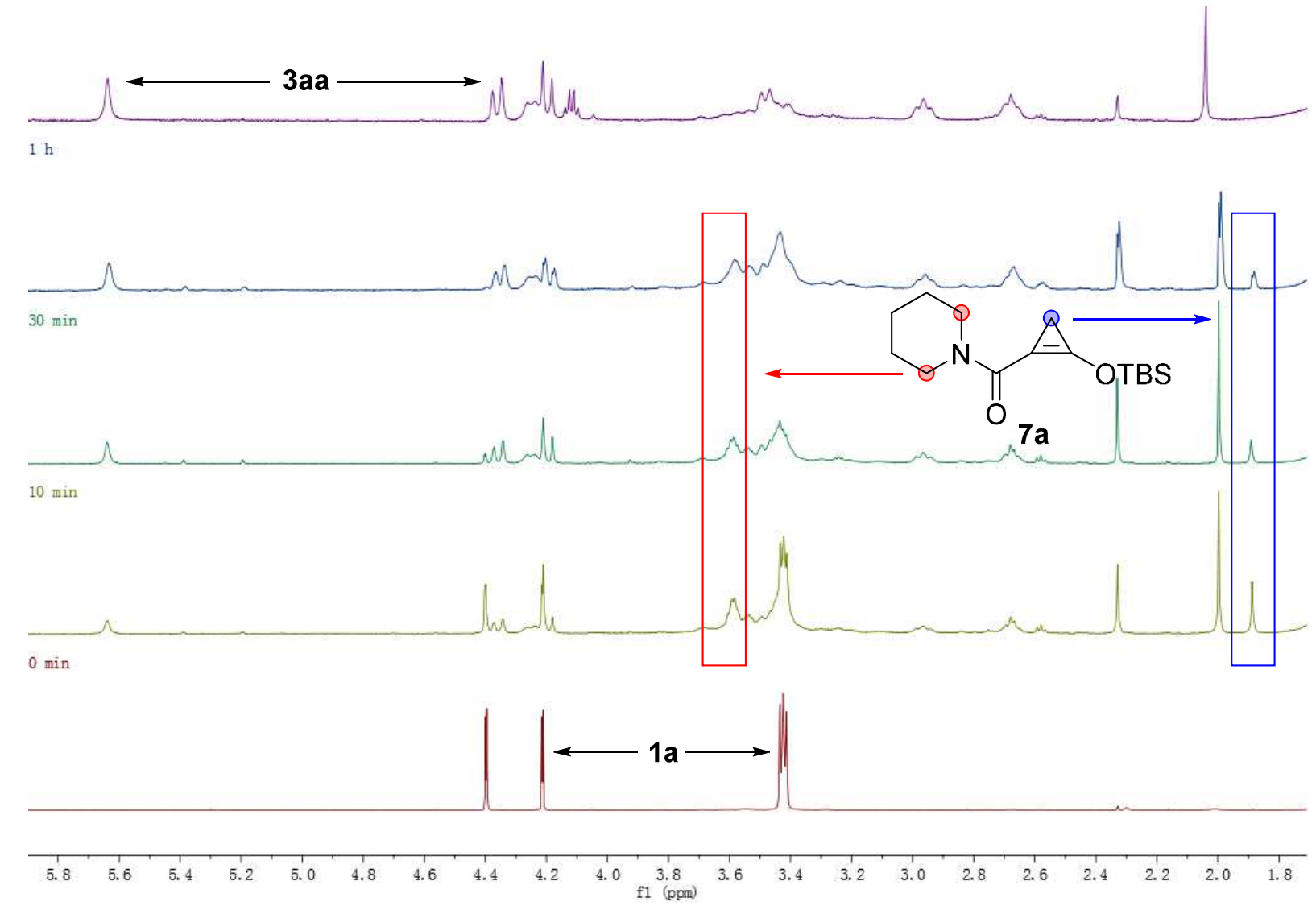

Scheme S1. ${ }^{1}$ H NMR spectral evidence for cyclopropene formation during copper-catalyzed [3+ 3]-cycloaddition.

$\mathrm{Cu}(\mathrm{MeCN})_{4} \mathrm{BF}_{4}(3.1 \mathrm{mg}, 0.01 \mathrm{mmol}, 5 \mathrm{~mol} \%)$, ligand 6 (5.8 mg, $\left.0.012 \mathrm{mmol}, 6 \mathrm{~mol} \%\right), 4 \AA$ molecular sieves $\left(50 \mathrm{mg}\right.$, activated), and $\operatorname{dry} \mathrm{CDCl}_{3}(2 \mathrm{~mL})$ were sequentially introduced into an oven-dried Schlenk tube under a flow of dry nitrogen. The resulting mixture was stirred at room temperature under the nitrogen atmosphere for $2 \mathrm{~h}$. Then nitrone $\mathbf{2 a}(39 \mathrm{mg}, 0.2 \mathrm{mmol})$ and enoldiazoacetamide 1a $(74 \mathrm{mg}, 0.24 \mathrm{mmol})$ were sequentially introduced into the system, and stirring was continued at room temperature. Part of the reaction mixure $(0.3 \mathrm{~mL})$ was transferred into an oven-dried NMR spectroscopy tube under a flow of dry nitrogen at specified times, and ${ }^{1} \mathrm{H}$ NMR spectra were recorded on an Agilent DD2-500 spectrometer (500 MHz). 


\section{2a (0.8 equiv)

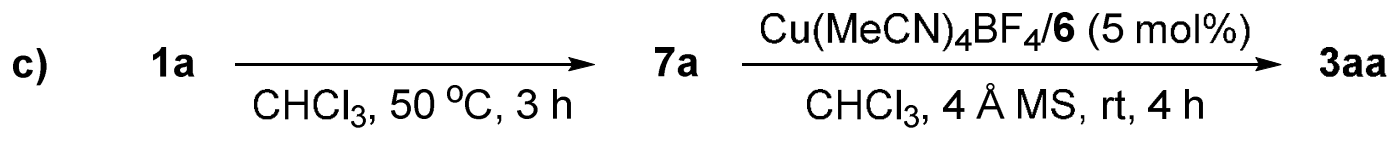

Enoldiazoacetamide 1a (74 mg, $0.24 \mathrm{mmol})$ and dry chloroform $(1 \mathrm{~mL})$ were introduced into an oven-dried Schlenk tube under a flow of dry nitrogen. The resulting solution was stirred at $50{ }^{\circ} \mathrm{C}$ under the nitrogen atmosphere for $3 \mathrm{~h}$, furnishing a solution of cyclopropene $7 \mathbf{a}$. $\mathrm{Cu}(\mathrm{MeCN})_{4} \mathrm{BF}_{4}(3.1 \mathrm{mg}, 0.01$ mmol, $5 \mathrm{~mol} \%$ ), ligand 6 (5.8 mg, $0.012 \mathrm{mmol}, 6 \mathrm{~mol} \%$ ), $4 \AA$ molecular sieves (50 mg, activated), and dry chloroform $(1 \mathrm{~mL})$ were sequentially introduced into an oven-dried Schlenk tube under a flow of dry nitrogen. The resulting mixture was stirred at room temperature under the nitrogen atmosphere for $2 \mathrm{~h}$. Then nitrone $\mathbf{2 a}$ (39 $\mathrm{mg}, 0.2 \mathrm{mmol})$ and the solution of cyclopropene $7 \mathbf{a}$ were sequentially introduced into the system, and stirring was continued at room temperature for $4 \mathrm{~h}$. Subsequently, the reaction mixture was concentrated in vacuo, and the residue was purified by flash chromatography on silica gel using hexanes/ethyl acetate as eluent to afford [3 + 3]-cycloaddition product 3aa in 93\% yield with $98 \%$ ee.

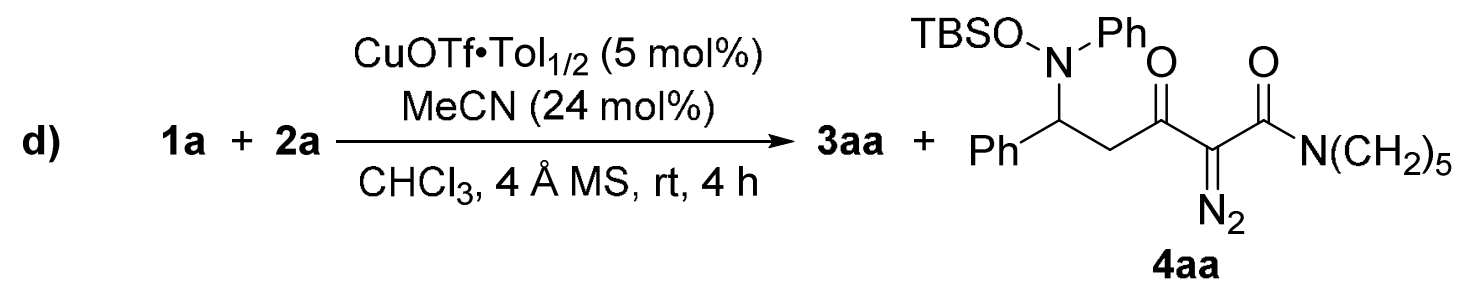

CuOTf $\cdot \mathrm{Tol}_{1 / 2}(2.6 \mathrm{mg}, 0.01 \mathrm{mmol}, 5 \mathrm{~mol} \%), 4 \AA$ molecular sieves (50 mg, activated), dry chloroform $(2 \mathrm{~mL}$ ), and dry acetonitrile (MeCN, $2.5 \mu \mathrm{L}, 0.048 \mathrm{mmol}, 24 \mathrm{~mol} \%$ ) were sequentially introduced into an oven-dried Schlenk tube under a flow of dry nitrogen. The resulting mixture was stirred at room temperature under the nitrogen atmosphere for $2 \mathrm{~h}$. Then nitrone 2a $(39 \mathrm{mg}, 0.2 \mathrm{mmol})$ and enoldiazoacetamide $1 \mathrm{a}(74 \mathrm{mg}, 0.24 \mathrm{mmol})$ were sequentially introduced into the system, and stirring was continued at room temperature for $4 \mathrm{~h}$. Subsequently, the reaction mixture was concentrated in vacuo, and the residue was purified by flash chromatography on silica gel using hexanes/ethyl acetate as eluent to afford [3 + 3]-cycloaddition product 3aa and Mannich addition product 4aa in 33\% and 53\% yields, respectively. 


\section{X-Ray Diffraction Analysis for Compound 3ab}

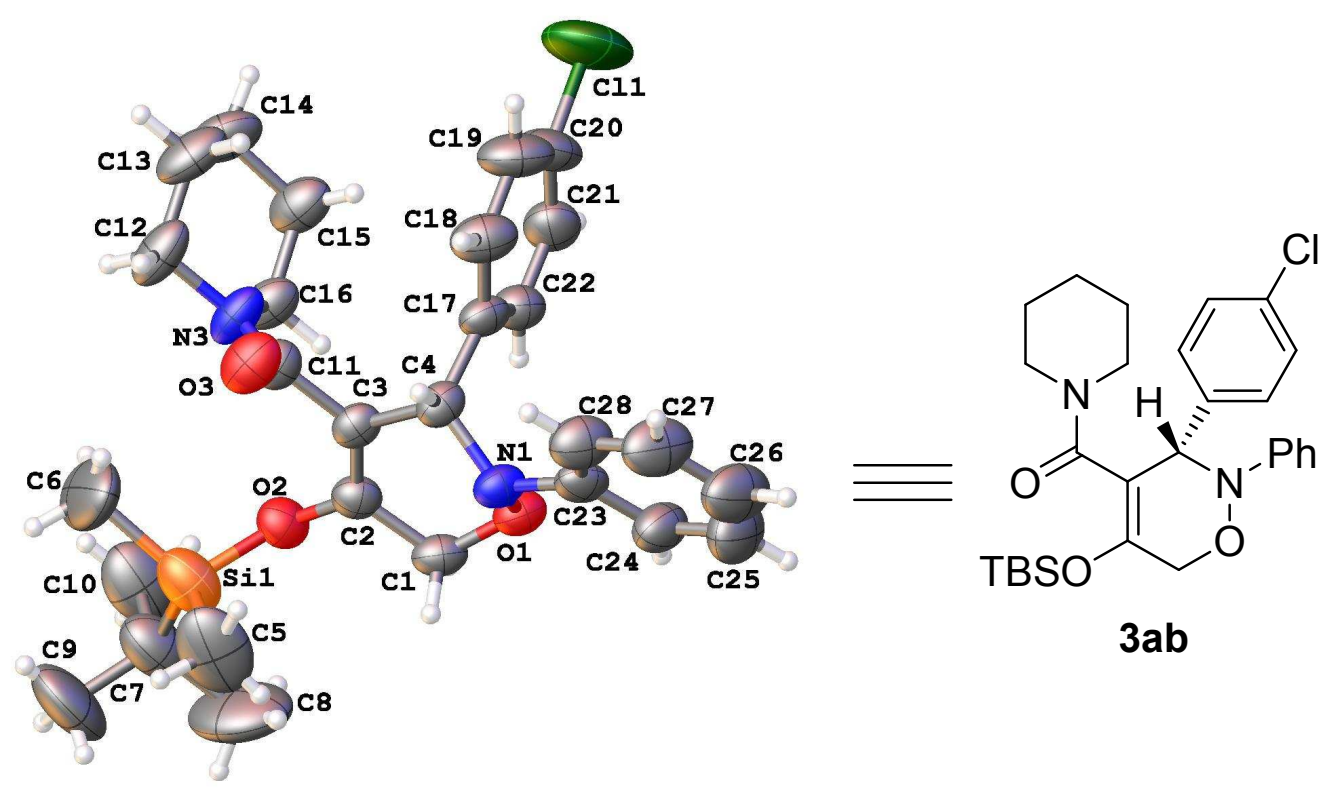

Figure S1. Single-crystal structure of $(S)$-[5-(tert-Butyldimethylsilyloxy)-3-(4-chlorophenyl)-2-phenyl3,6-dihydro-2H-1,2-oxazin-4-yl](piperidin-1-yl)methanone (3ab). CCDC 1417945 contains supplementary crystallographic data for $\mathbf{3 a b}$.

Single crystals of $\mathrm{C}_{28} \mathrm{H}_{37} \mathrm{ClN}_{2} \mathrm{O}_{3} \mathrm{Si}$ were prepared by slow evaporation of hexanes. A suitable colorless plate-like crystal, with dimensions of $0.53 \mathrm{~mm} \times 0.37 \mathrm{~mm} \times 0.07 \mathrm{~mm}$, was mounted in epoxy onto a glass fiber. The data were collected at 293(2) K using a Rigaku AFC12 / Saturn 724 CCD fitted with MoK $\alpha$ radiation $(\lambda=0.71075 \AA)$. Data collection and unit cell refinement were performed using CrystalClear software. ${ }^{4}$ The total number of data were measured in the range $5.6^{\circ}<2 \theta<50.1^{\circ}$ using $\omega$ scans. Data processing and absorption correction, giving minimum and maximum transmission factors $(0.308,1.000)$, were accomplished with CrystalClear ${ }^{4}$ and $A B S C O R^{5}$, respectively. The structure, using Olex $2^{6}$, was solved with the ShelXT ${ }^{7}$ structure solution program using direct methods and refined (on $F^{2}$ ) with the ShelXL ${ }^{8}$ refinement package using full-matrix, least-squares techniques. All non-hydrogen atoms were refined with anisotropic displacement parameters. All hydrogen atom positions were determined by geometry and refined by a riding model. 
Table S3. Crystallographic Data and Structure Refinement for 3ab

\begin{tabular}{|c|c|}
\hline Identification code & cd1356a \\
\hline Empirical formula & $\mathrm{C}_{28} \mathrm{H}_{37} \mathrm{ClN}_{2} \mathrm{O}_{3} \mathrm{Si}$ \\
\hline Formula weight & 513.13 \\
\hline Crystal system & Monoclinic \\
\hline Space group & $P 1211$ \\
\hline$a(\AA)$ & $6.520(4)$ \\
\hline$b(\AA)$ & $18.360(10)$ \\
\hline$c(\AA \dot{A})$ & $12.283(7)$ \\
\hline$\alpha\left(^{\circ}\right)$ & 90 \\
\hline$\beta\left(^{\circ}\right)$ & $99.174(10)$ \\
\hline$\gamma\left({ }^{\circ}\right)$ & 90 \\
\hline Volume $\left(\AA^{3}\right)$ & $1451.5(15)$ \\
\hline $\mathrm{Z}$ & 2 \\
\hline$\rho$ (calc.) & 1.174 \\
\hline$\lambda$ & 0.71075 \\
\hline Temp. (K) & $293(2)$ \\
\hline $\mathrm{F}(000)$ & 548 \\
\hline$\mu\left(\mathrm{mm}^{-1}\right)$ & 0.203 \\
\hline $\mathrm{T}_{\min }, \mathrm{T}_{\max }$ & $0.308,1.000$ \\
\hline $2 \theta_{\text {range }}\left({ }^{\circ}\right)$ & 5.6 to 50.1 \\
\hline Reflections collected & 7062 \\
\hline Independent reflections & $\begin{array}{c}3975 \\
{[\mathrm{R}(\mathrm{int})=0.0413]}\end{array}$ \\
\hline Completeness & $99.2 \%$ \\
\hline Data / restraints / parameters & 3975 / 2 / 321 \\
\hline $\begin{array}{c}\text { Observed data } \\
{[\mathrm{I}>2 \sigma(\mathrm{I})]}\end{array}$ & 3205 \\
\hline$w R\left(F^{2}\right.$ all data $)$ & 0.1474 \\
\hline$R(F$ obsd data $)$ & 0.0782 \\
\hline Goodness-of-fit on $F^{2}$ & 1.052 \\
\hline largest diff. peak and hole (e $\AA^{-3}$ ) & $1.17 /-0.54$ \\
\hline
\end{tabular}

$$
\begin{gathered}
w R_{2}=\left\{\Sigma\left[w\left(F_{\mathrm{o}}^{2}-F_{\mathrm{c}}^{2}\right)^{2}\right] / \Sigma\left[w\left(F_{\mathrm{o}}^{2}\right)^{2}\right]\right\}^{1 / 2} \\
R_{1}=\Sigma \| F_{\mathrm{o}}|-| F_{\mathrm{c}}|/ \Sigma| F_{\mathrm{o}} \mid
\end{gathered}
$$




\section{References}

(1) (a) Deng, Y.; Jing, C.; Doyle, M. P. Chem. Commun. 2015, 51, 12924. (b) Davies, H. M. L.; Ahmed, G.; Churchill, M. R. J. Am. Chem. Soc. 1996, 118, 10774.

(2) (a) Lo, M. M.-C.; Fu, G. C. J. Am. Chem. Soc. 2002, 124, 4572. (b) Murahashi, S.-I.; Mitsui, H.; Shiota, T.; Tsuda, T.; Watanabe, S. J. Org. Chem. 1990, 55, 1736. (c) West, P. R.; Davis, G. C. J. Org. Chem. 1989, 54, 5176.

(3) Armarego, W. L. F.; Chai, C. L. L. Purification of Laboratory Chemicals, 6th ed.; Elsevier Inc.: London, 2009.

(4) CrystalClear User's Manual; Rigaku/MSC Inc., Rigaku Corporation: the Woodlands, TX, 2011.

(5) Higashi, T. ABSCOR; Rigaku Corporation: Tokyo, Japan, 1995.

(6) Dolomanov, O. V.; Bourhis, L. J.; Gildea, R. J.; Howard, J. A. K.; Puschmann, H. J. Appl. Cryst. 2009, 42, 339.

(7) Sheldrick, G. M. Acta Cryst. 2015, A71, 3.

(8) Sheldrick, G. M. Acta Cryst. 2008, A64, 112. 


\section{NMR Spectra for Compounds 3 and 4}

[5-(tert-Butyldimethylsilyloxy)-2,3-diphenyl-3,6-dihydro-2H-1,2-oxazin-4-yl](piperidin-1-yl)methanone (3aa)

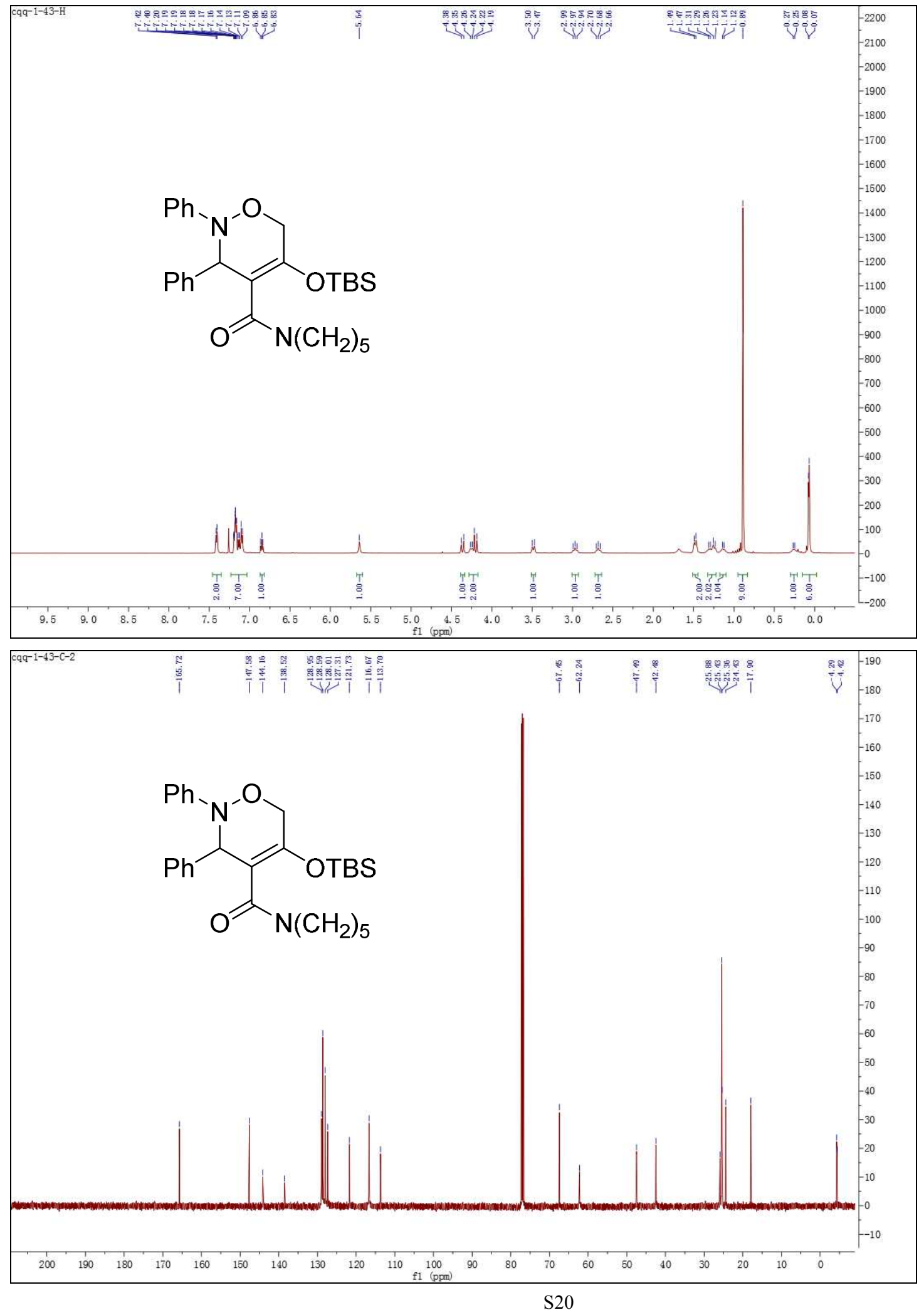


[5-(tert-Butyldimethylsilyloxy)-3-(4-chlorophenyl)-2-phenyl-3,6-dihydro-2H-1,2-oxazin-4-yl](piperidin-1-yl)methanone (3ab)
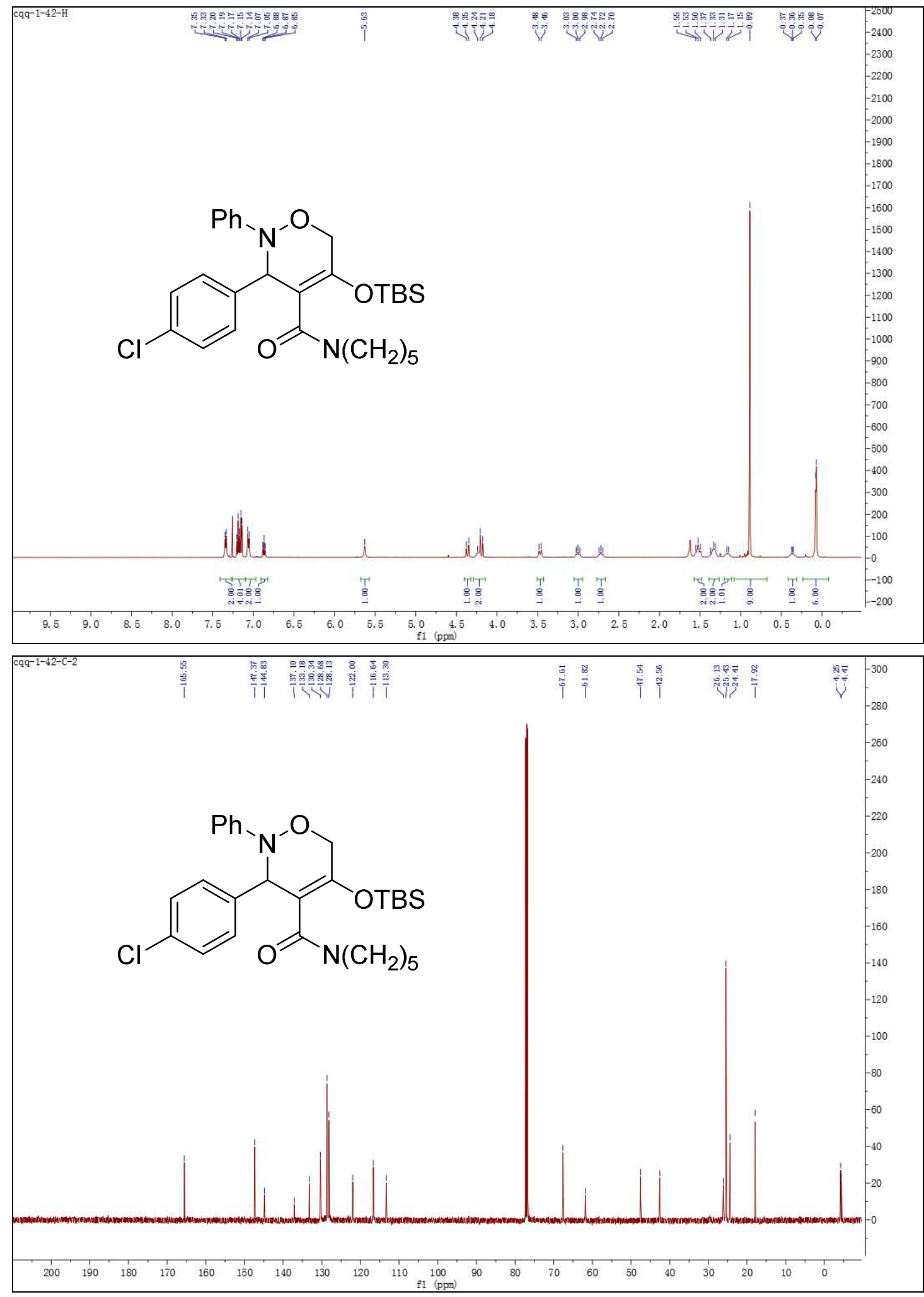
[5-(tert-Butyldimethylsilyloxy)-2-phenyl-3-(p-tolyl)-3,6-dihydro-2H-1,2-oxazin-4-yl](piperidin-1-yl) methanone (3ac)
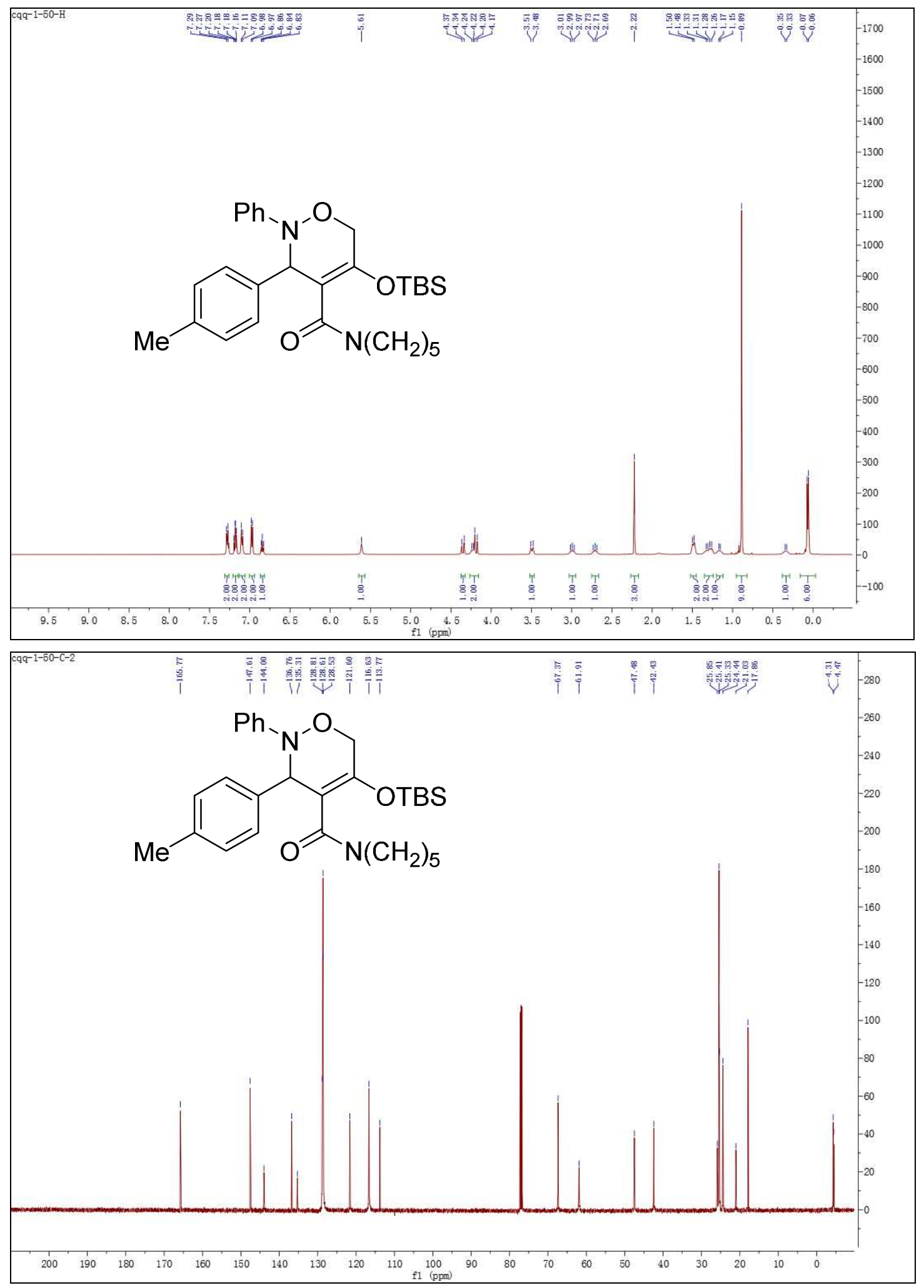
[5-(tert-Butyldimethylsilyloxy)-3-(4-methoxyphenyl)-2-phenyl-3,6-dihydro-2H-1,2-oxazin-4-yl](piperidin-1-yl)methanone (3ad)
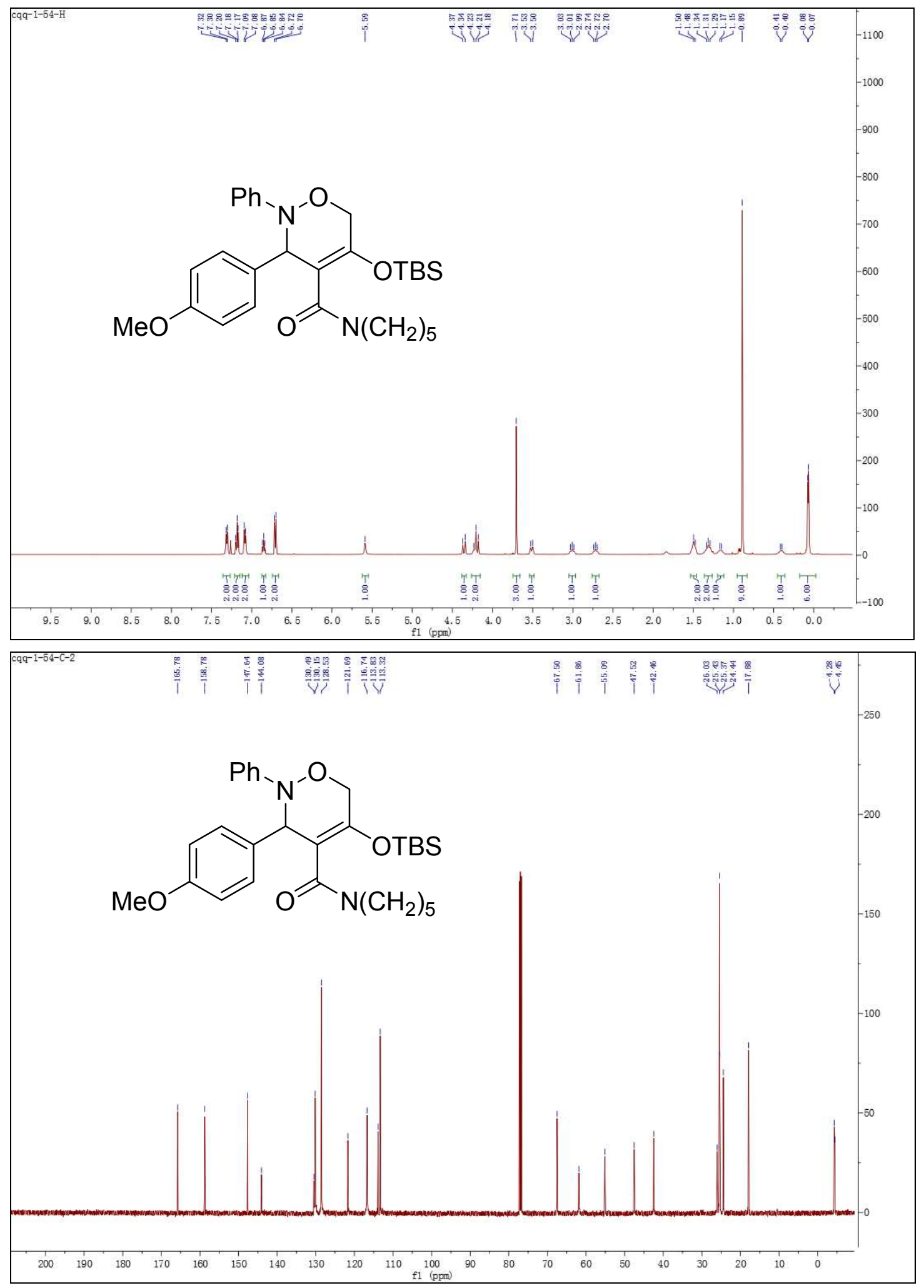
[5-(tert-Butyldimethylsilyloxy)-3-(3-chlorophenyl)-2-phenyl-3,6-dihydro-2H-1,2-oxazin-4-yl](piperidin-1-yl)methanone (3ae)
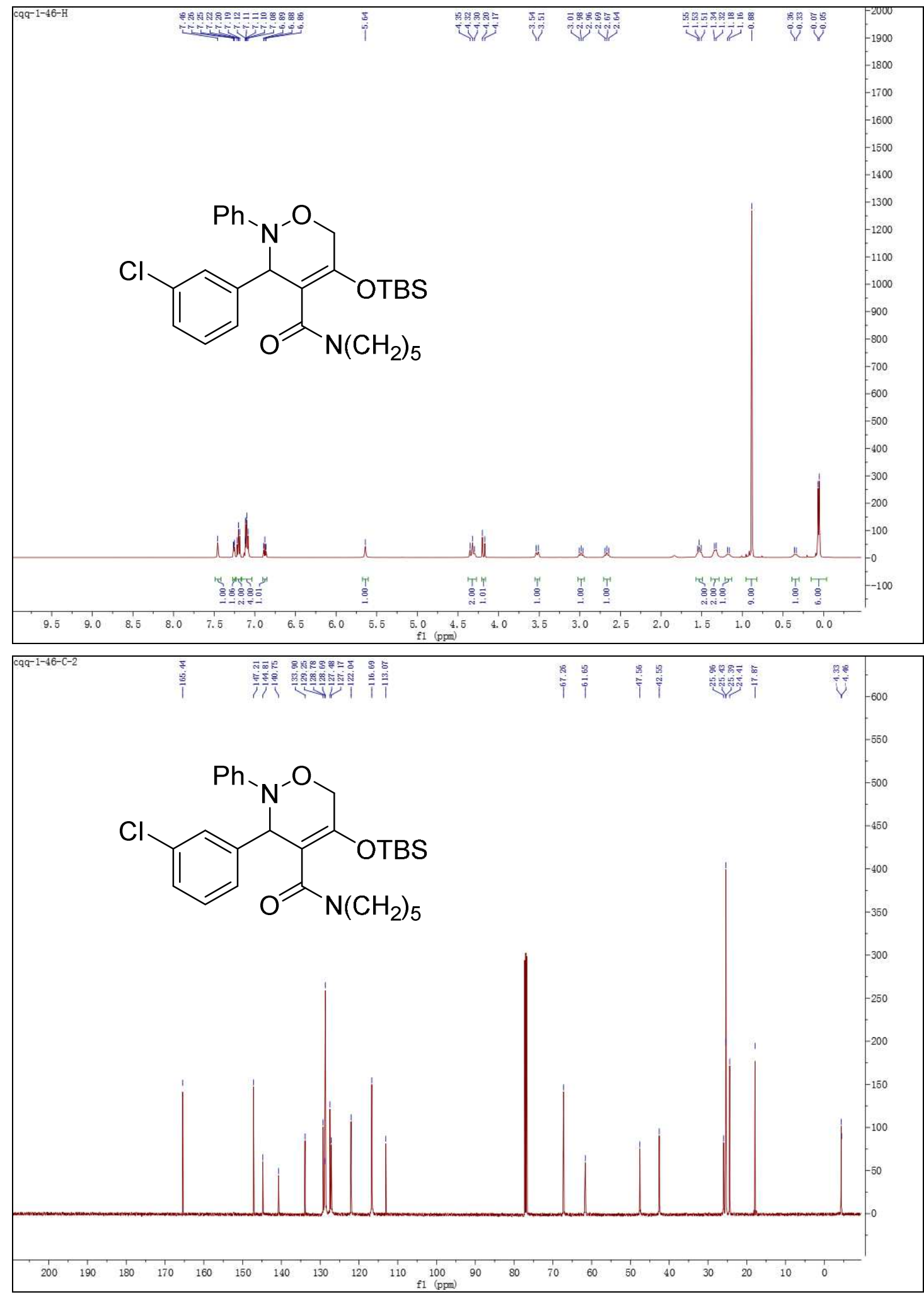
[5-(tert-Butyldimethylsilyloxy)-3-(naphthalen-2-yl)-2-phenyl-3,6-dihydro-2H-1,2-oxazin-4-yl](piperidin-1-yl)methanone (3af)

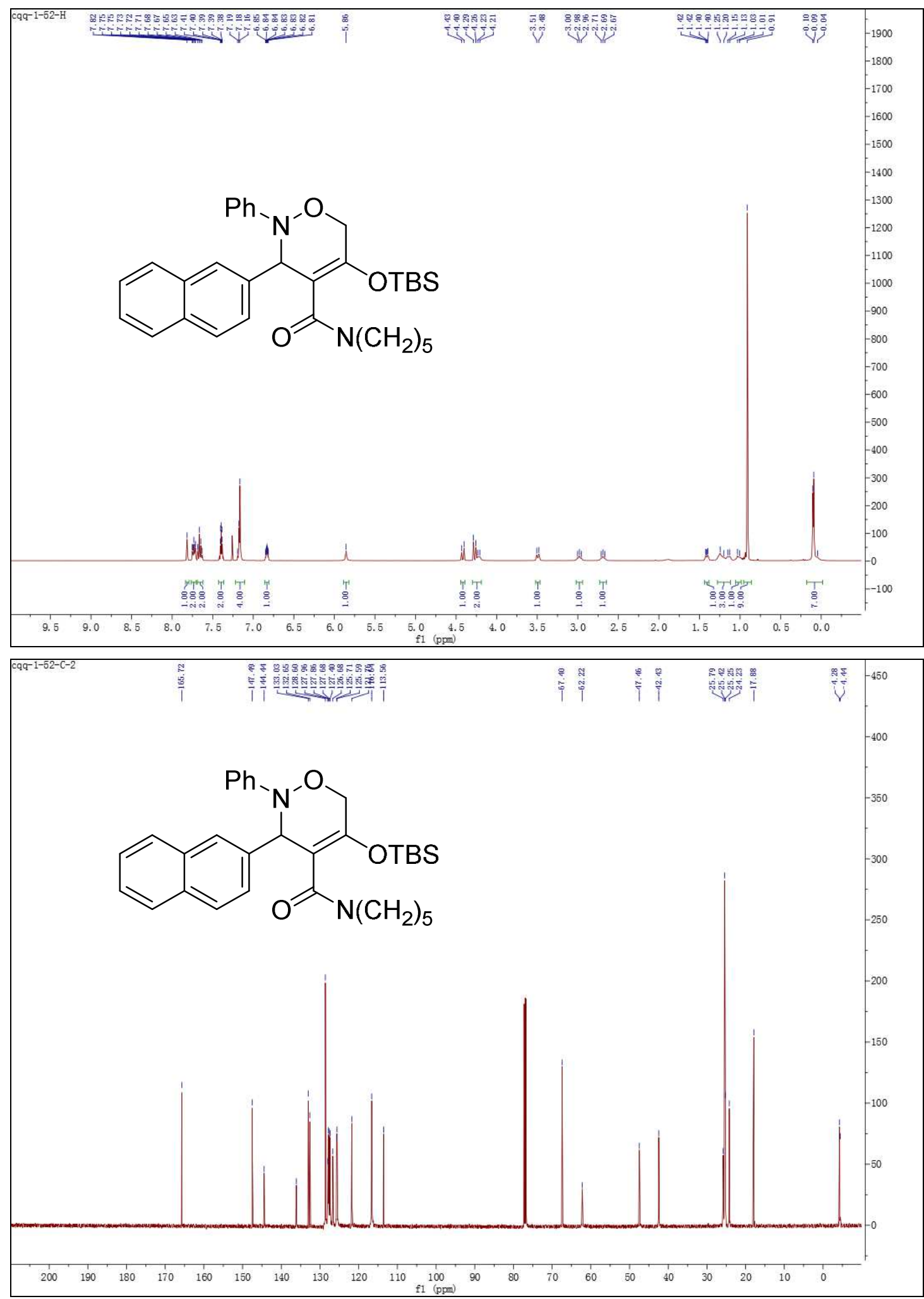


[5-(tert-Butyldimethylsilyloxy)-3-(furan-2-yl)-2-phenyl-3,6-dihydro-2H-1,2-oxazin-4-yl](piperidin1-yl)methanone (3ag)
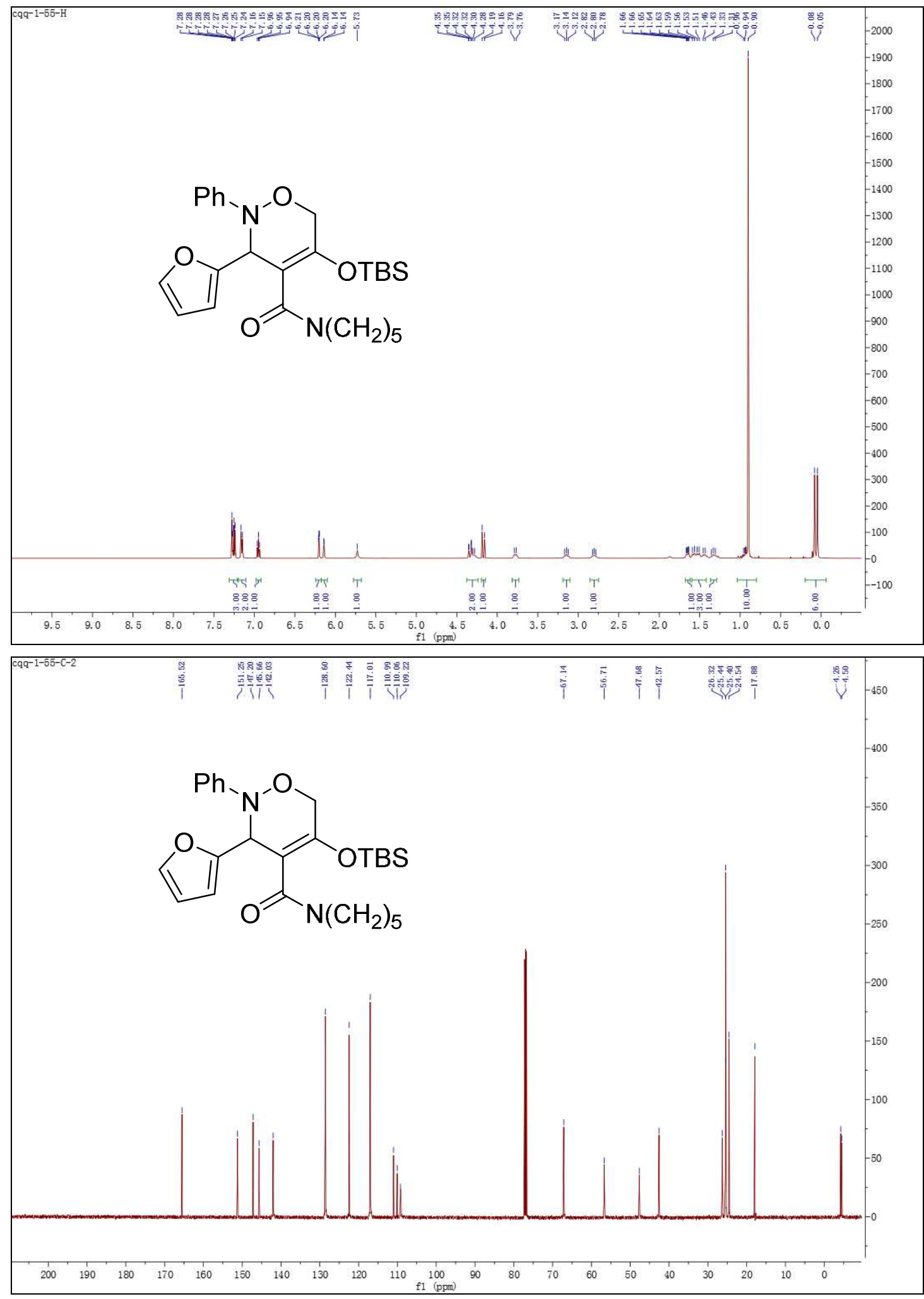
Ethyl 4-[5-(tert-Butyldimethylsilyloxy)-3-phenyl-4-(piperidine-1-carbonyl)-3,6-dihydro-2H-1,2oxazin-2-yl]benzoate (3ah)

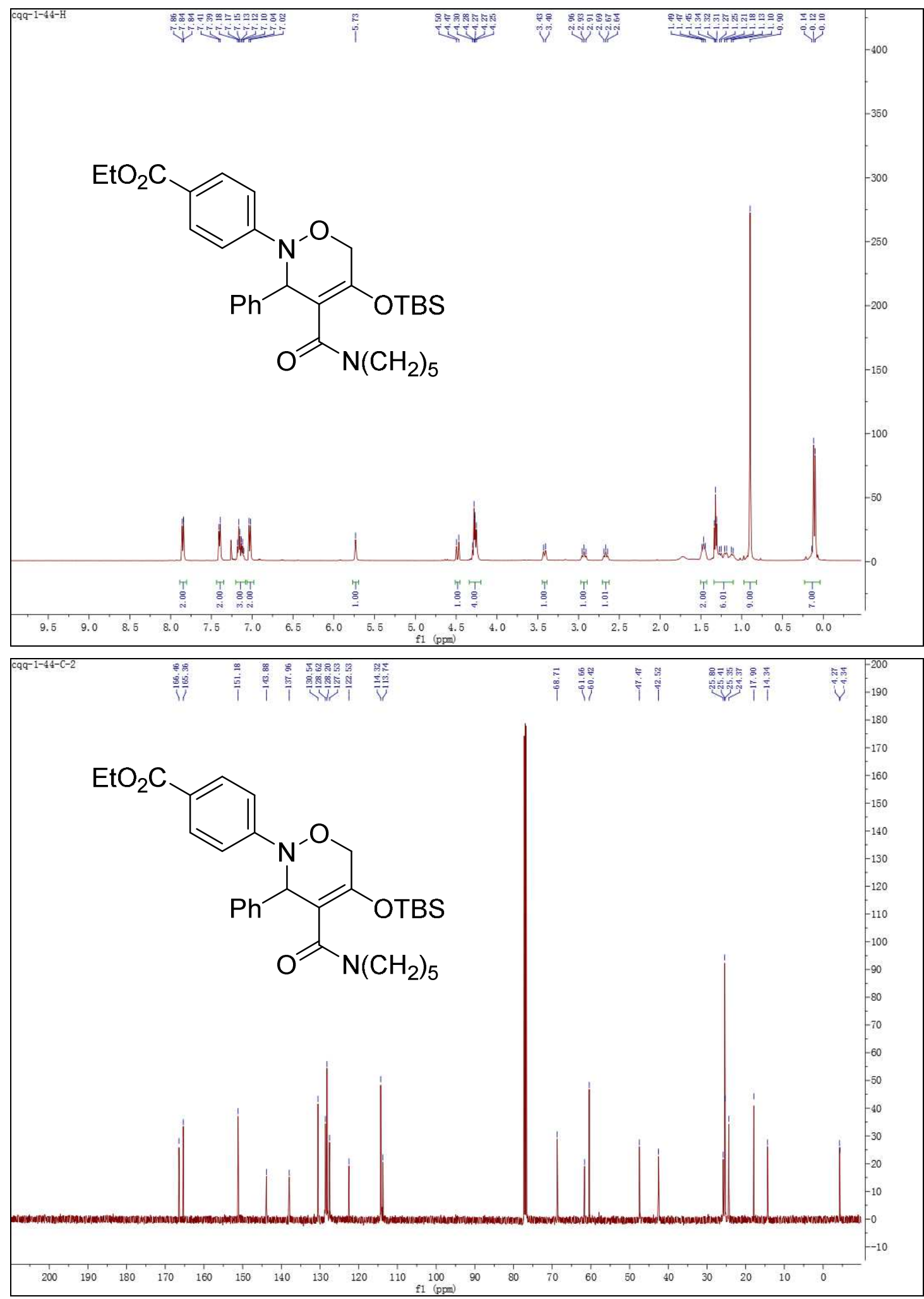


[2-(3-Bromo-4-methylphenyl)-5-(tert-butyldimethylsilyloxy)-3-phenyl-3,6-dihydro-2H-1,2-oxazin4-yl](piperidin-1-yl)methanone (3ai)

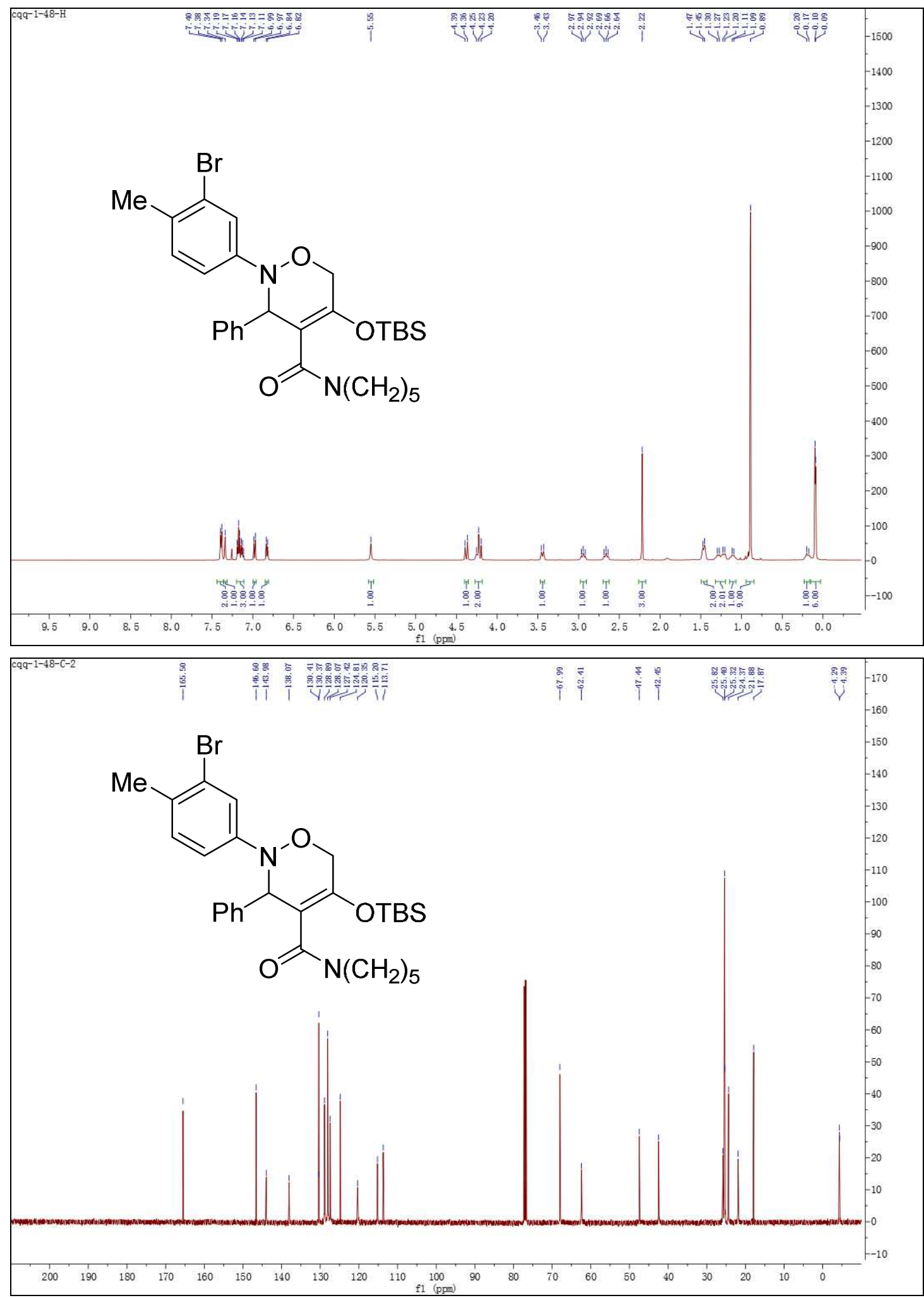


[2-Benzyl-5-(tert-butyldimethylsilyloxy)-3-cyclohexyl-3,6-dihydro-2H-1,2-oxazin-4-yl](piperidin-1yl)methanone (3aj)
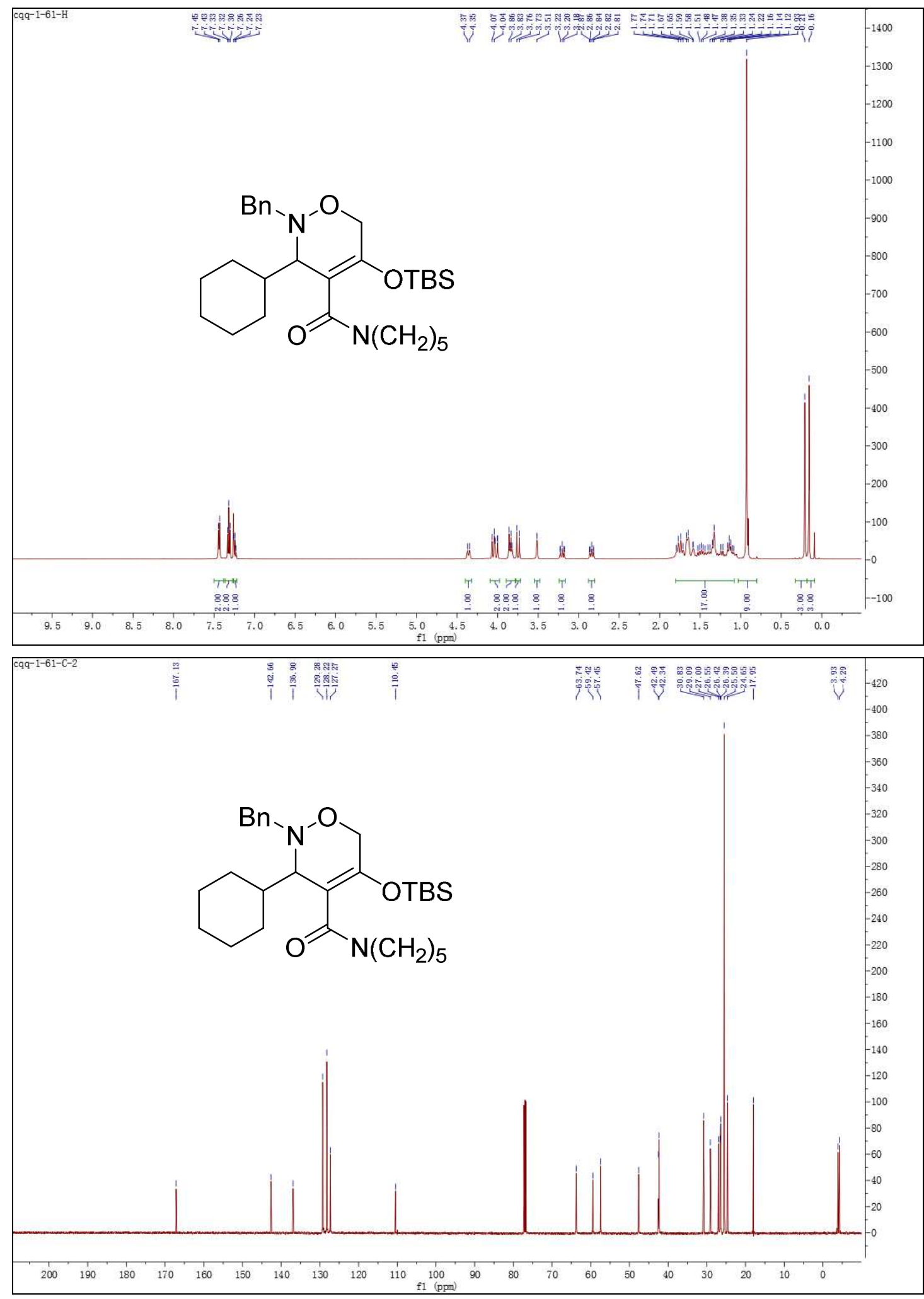
5-(tert-Butyldimethylsilyloxy)- $N, N$-dimethyl-2,3-diphenyl-3,6-dihydro-2H-1,2-oxazine-4-carboxamide (3ba)
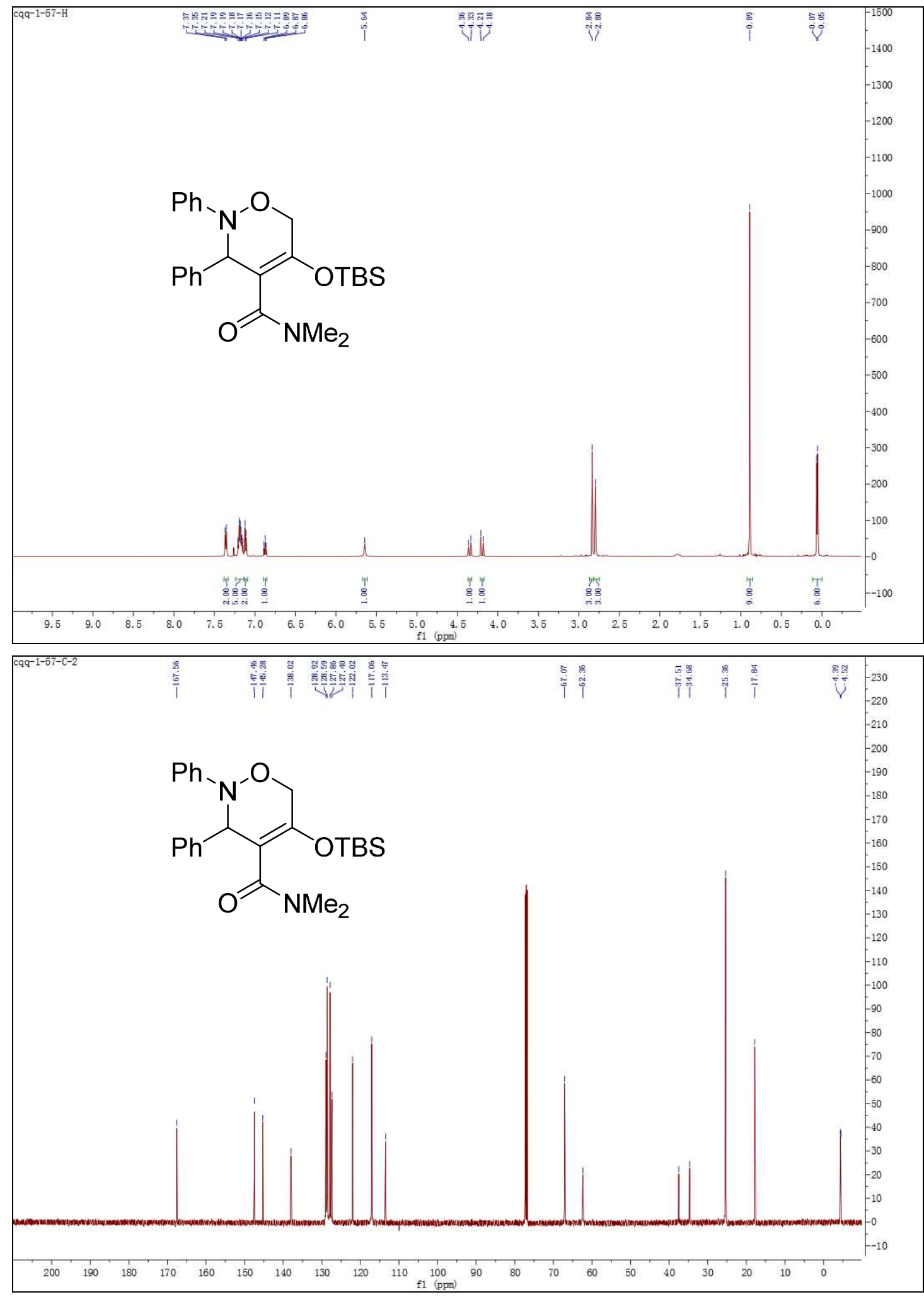
[5-(tert-Butyldimethylsilyloxy)-6-methyl-2,3-diphenyl-3,6-dihydro-2H-1,2-oxazin-4-yl](piperidin-1yl)methanone (3ca)
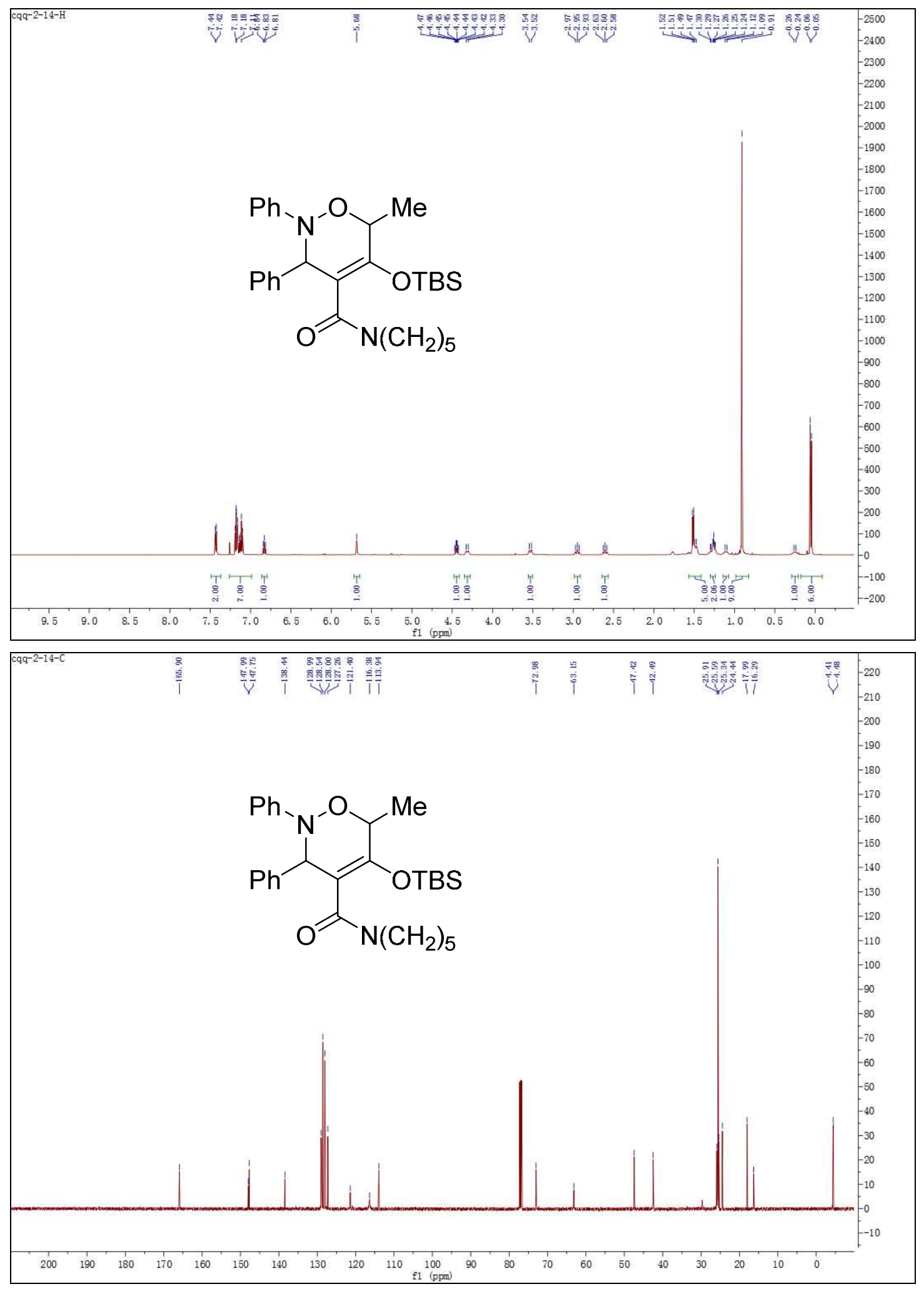
[5-(tert-Butyldimethylsilyloxy)-6-ethyl-2,3-diphenyl-3,6-dihydro-2H-1,2-oxazin-4-yl](piperidin-1yl)methanone (3da)

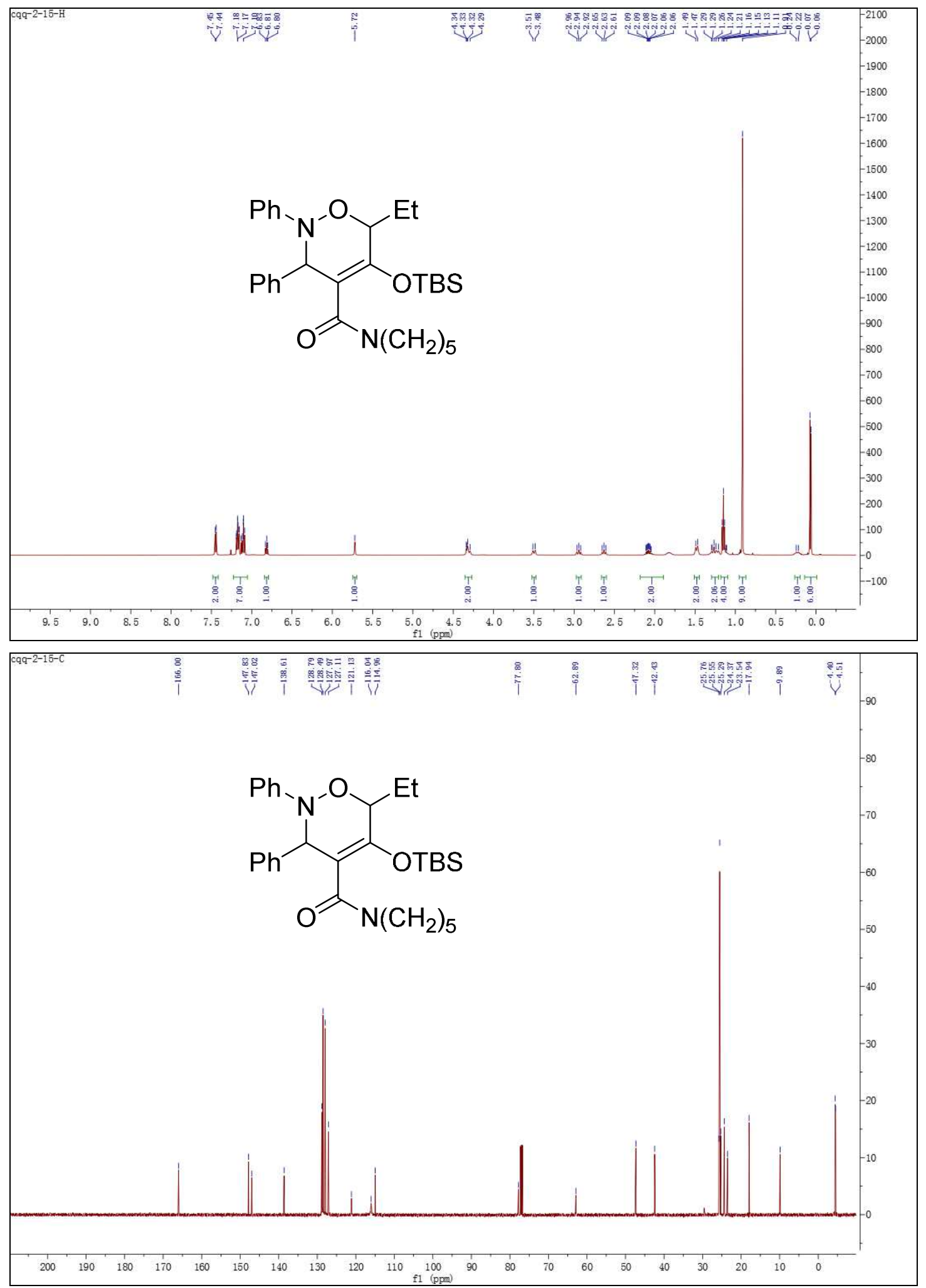


5-[(tert-Butyldimethylsilyloxy)(phenyl)amino]-2-diazo-5-phenyl-1-(piperidin-1-yl)pentane-1,3dione (4aa)

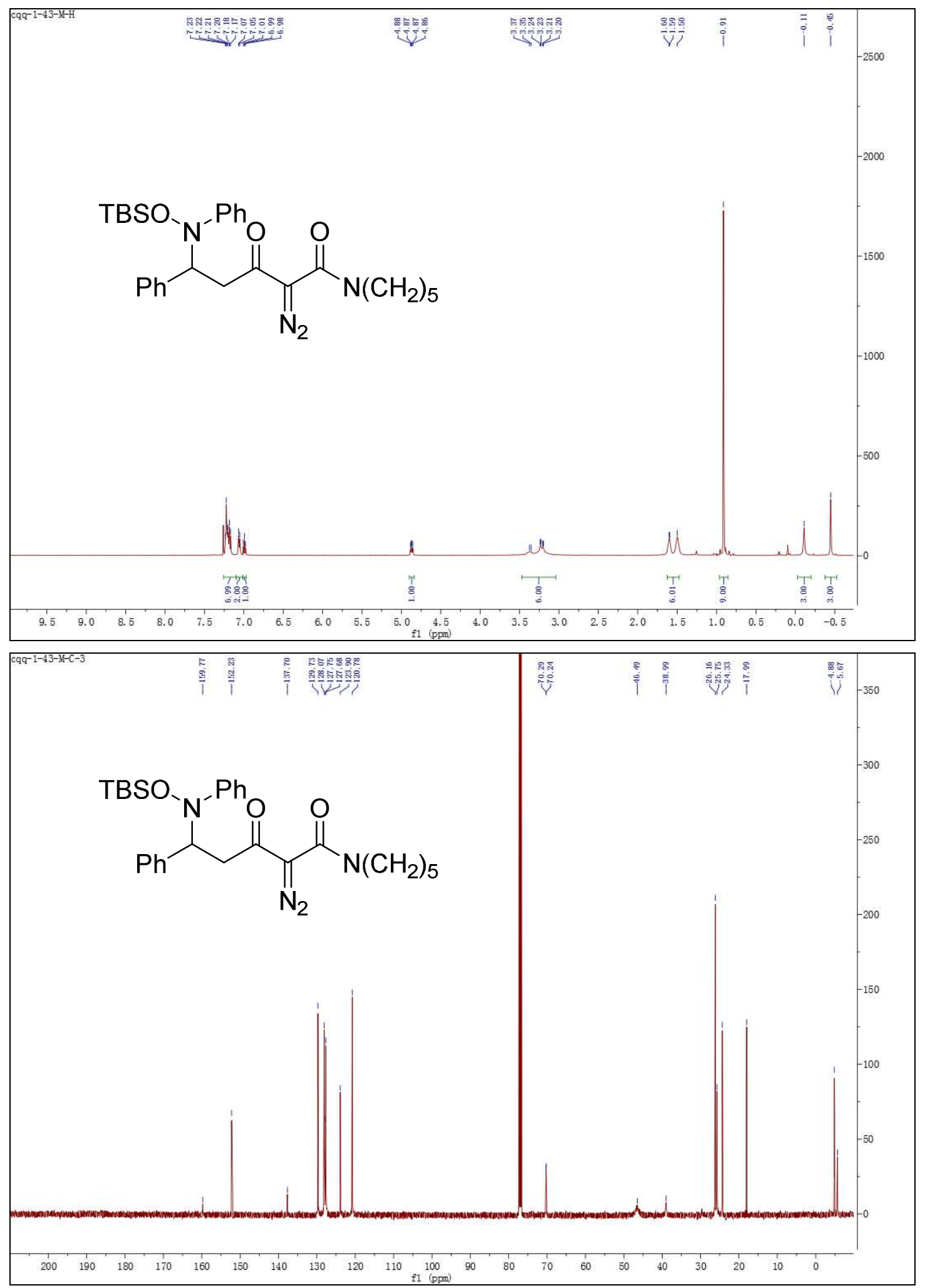


5-[(tert-Butyldimethylsilyloxy)(phenyl)amino]-5-(4-chlorophenyl)-2-diazo-1-(piperidin-1-yl)pentane-1,3-dione (4ab)

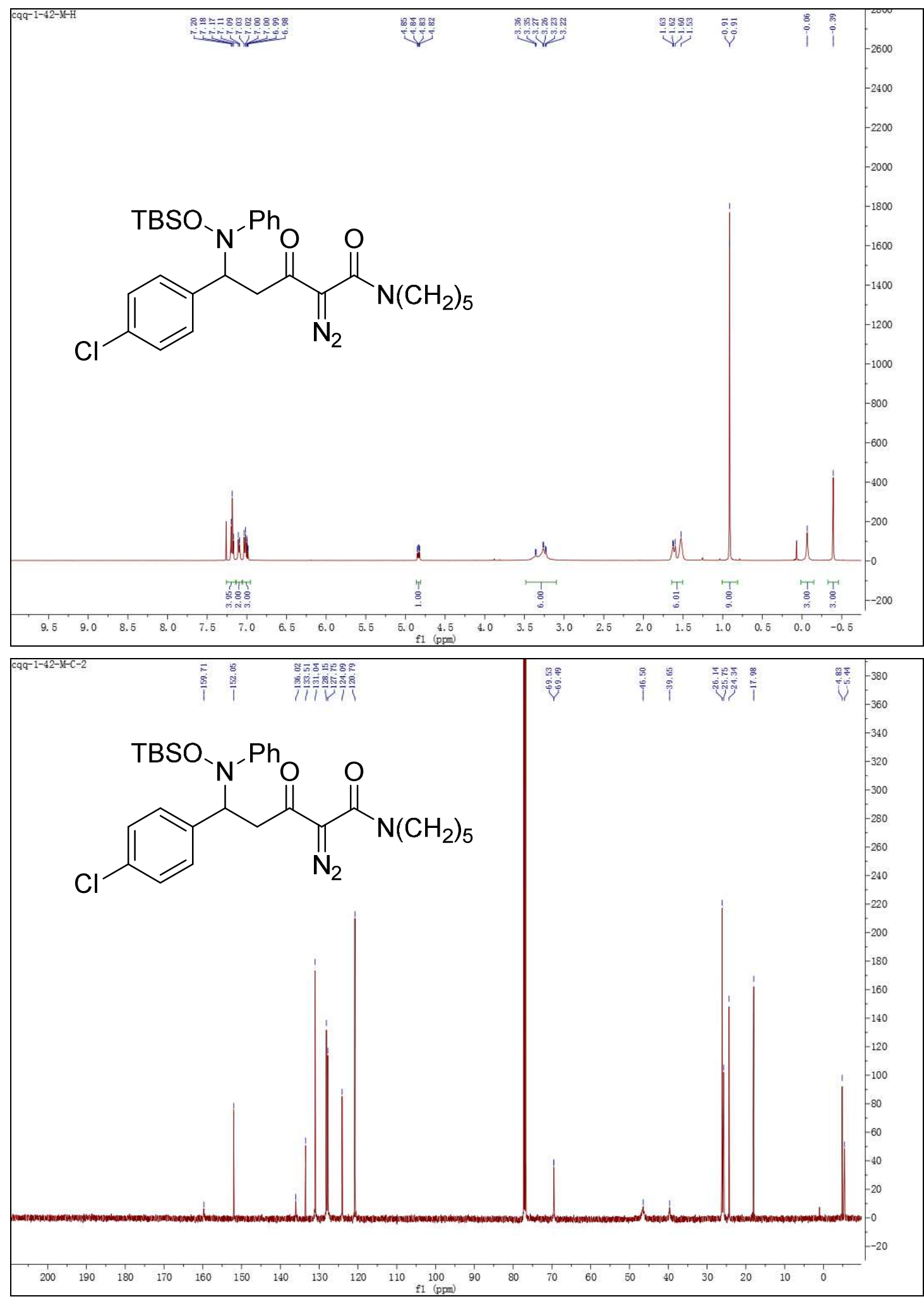


5-[(tert-Butyldimethylsilyloxy)(phenyl)amino]-2-diazo-5-(naphthalen-2-yl)-1-(piperidin-1-yl)pentane-1,3-dione (4af)

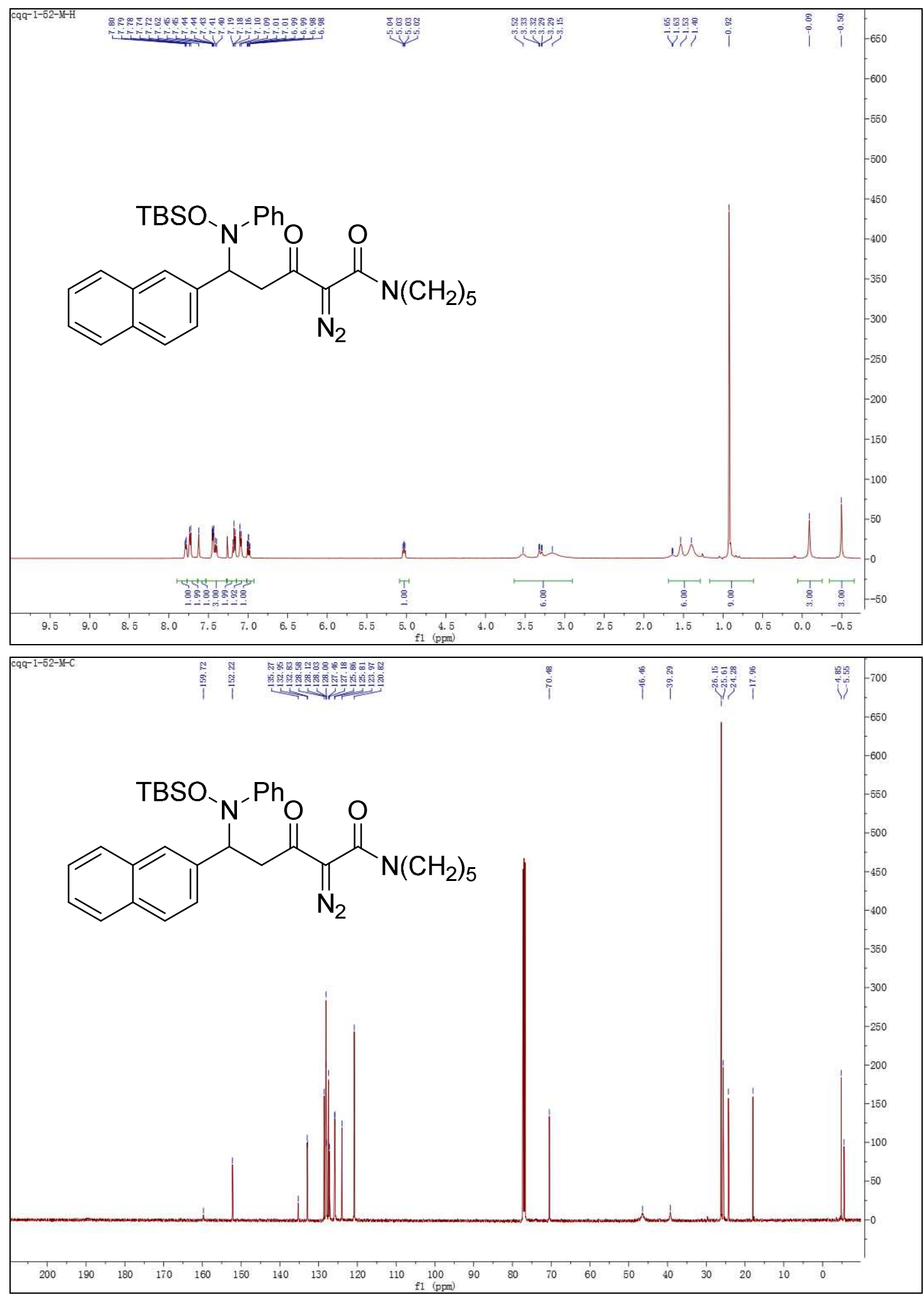


Ethyl 4-[(tert-Butyldimethylsilyloxy)(4-diazo-3,5-dioxo-1-phenyl-5-(piperidin-1-yl)pentyl)amino] benzoate (4ah)
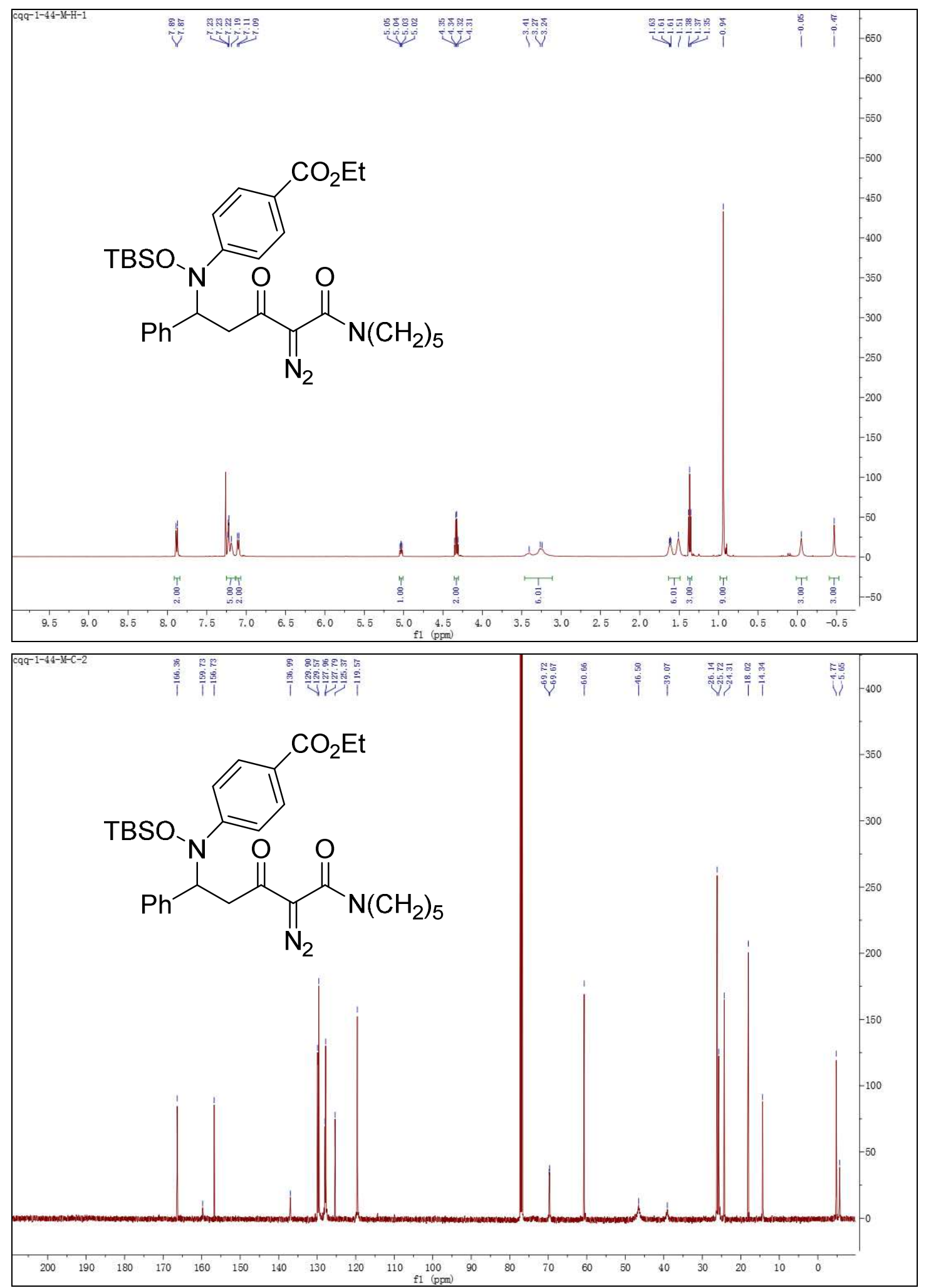


\section{HPLC Analyses for Compounds 3}

[5-(tert-Butyldimethylsilyloxy)-2,3-diphenyl-3,6-dihydro-2H-1,2-oxazin-4-yl](piperidin-1-yl)methanone (3aa)
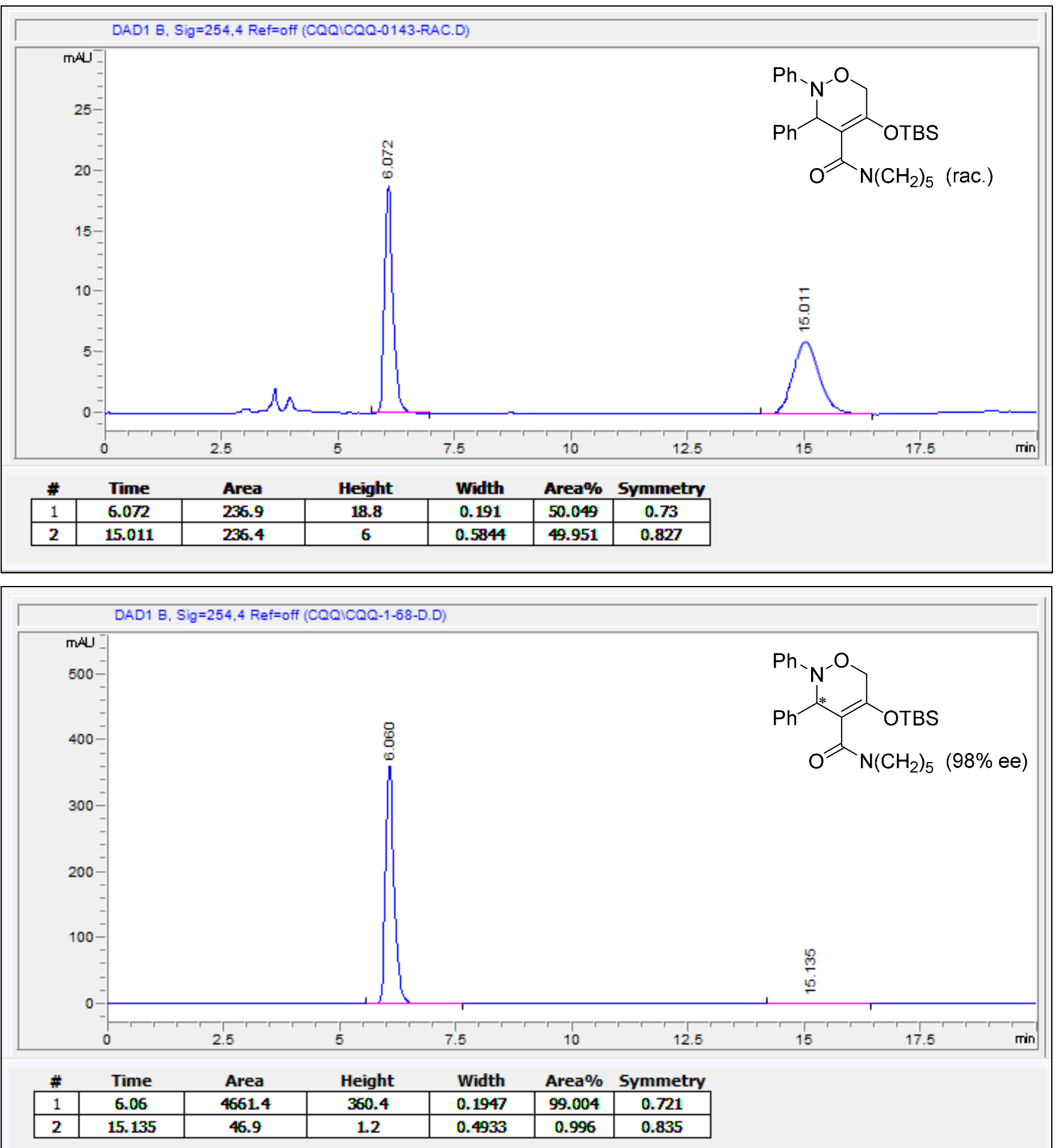
[5-(tert-Butyldimethylsilyloxy)-3-(4-chlorophenyl)-2-phenyl-3,6-dihydro-2H-1,2-oxazin-4-yl](piperidin-1-yl)methanone (3ab)
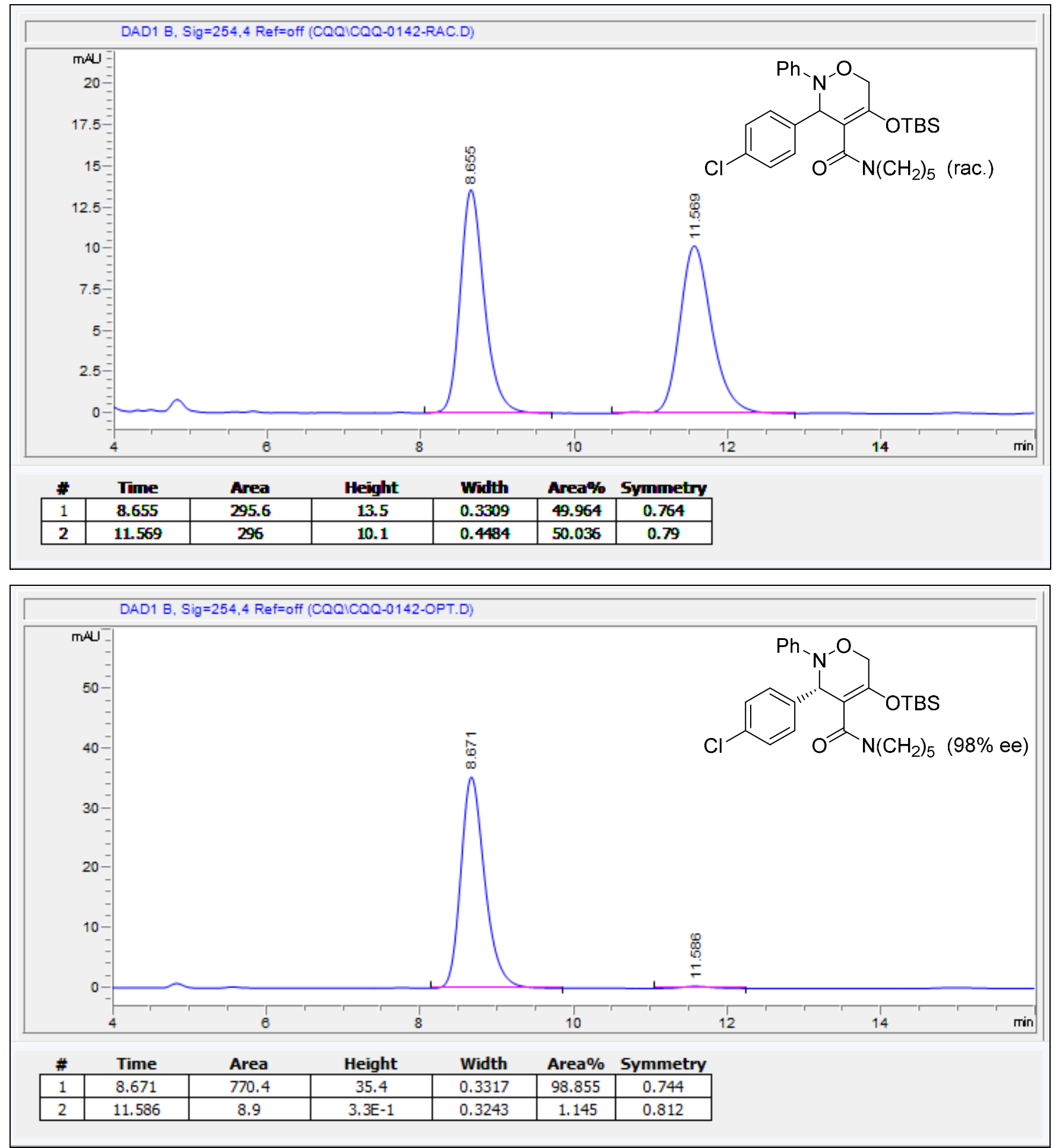
[5-(tert-Butyldimethylsilyloxy)-2-phenyl-3-(p-tolyl)-3,6-dihydro-2H-1,2-oxazin-4-yl](piperidin-1-yl) methanone (3ac)
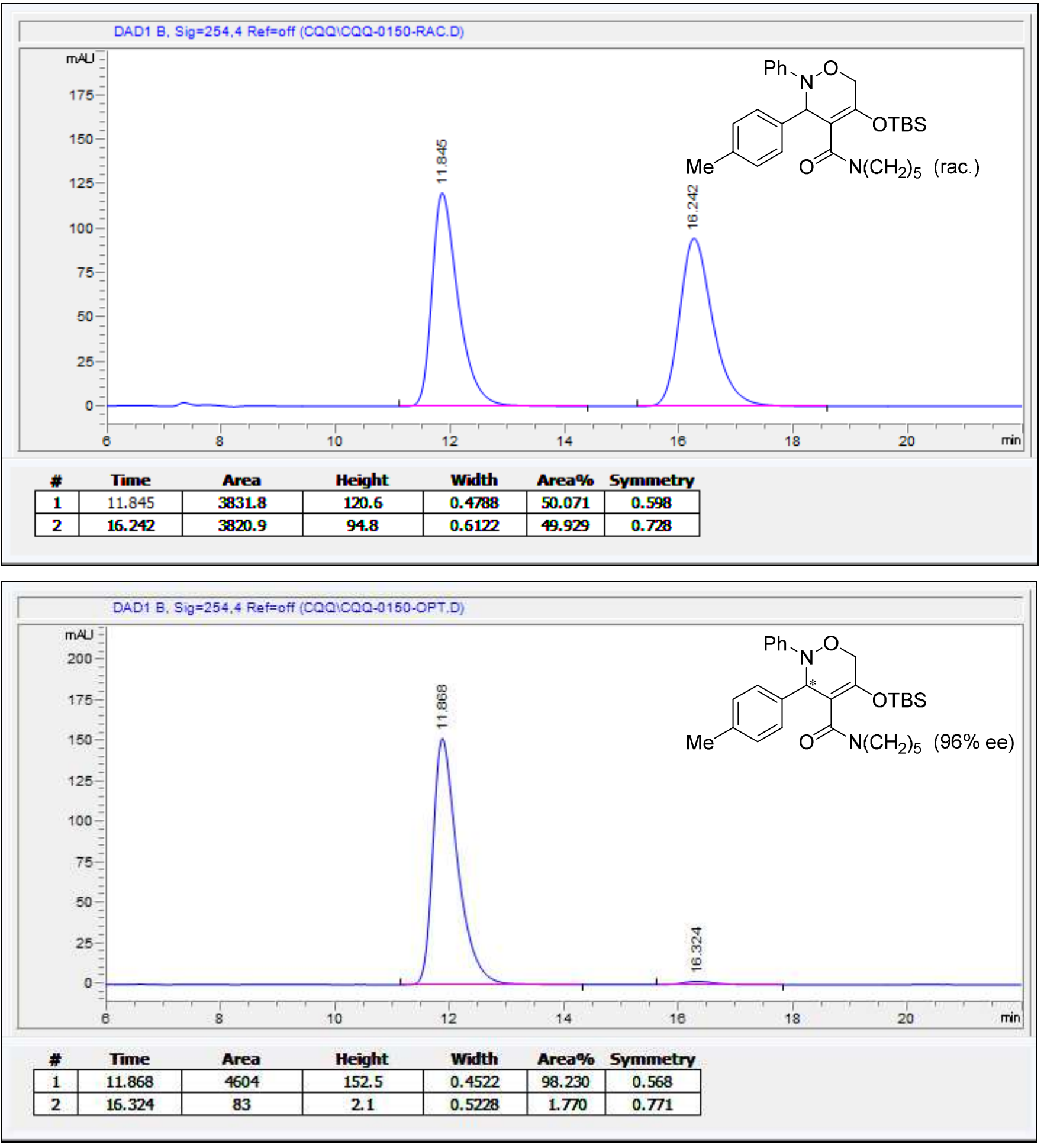
[5-(tert-Butyldimethylsilyloxy)-3-(4-methoxyphenyl)-2-phenyl-3,6-dihydro-2H-1,2-oxazin-4-yl](piperidin-1-yl)methanone (3ad)
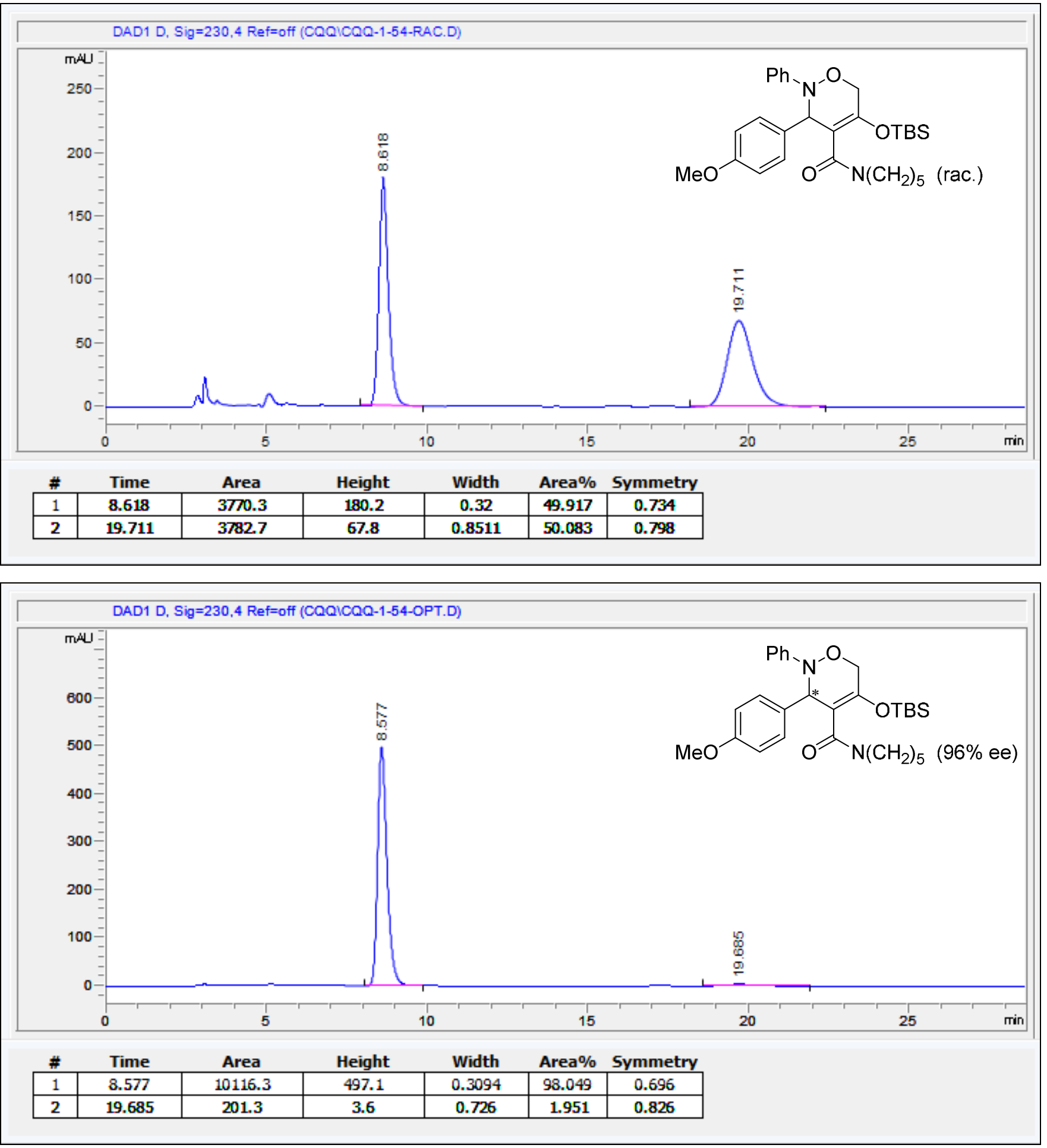
[5-(tert-Butyldimethylsilyloxy)-3-(3-chlorophenyl)-2-phenyl-3,6-dihydro-2H-1,2-oxazin-4-yl](piperidin-1-yl)methanone (3ae)
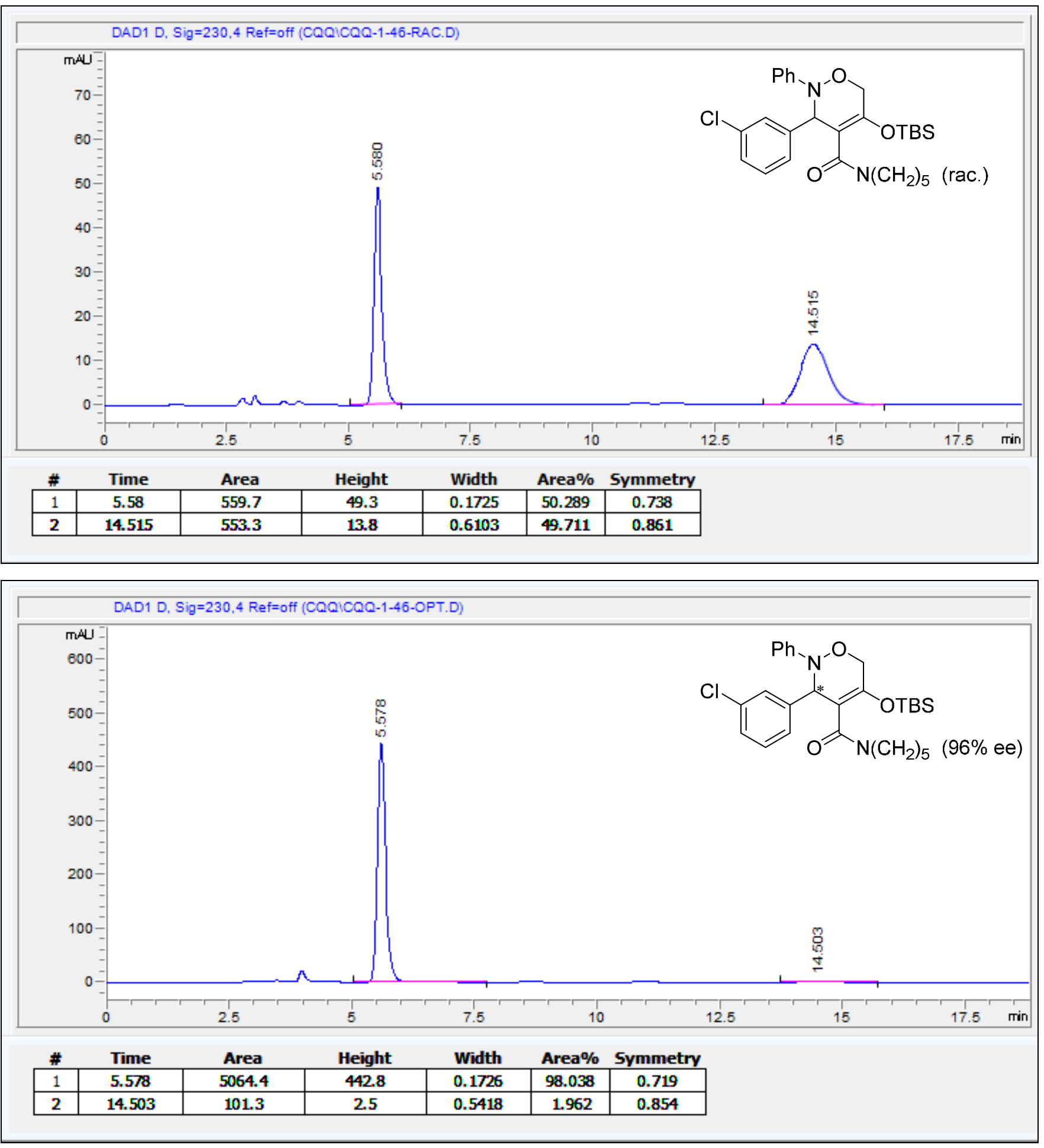
[5-(tert-Butyldimethylsilyloxy)-3-(naphthalen-2-yl)-2-phenyl-3,6-dihydro-2H-1,2-oxazin-4-yl](piperidin-1-yl)methanone (3af)
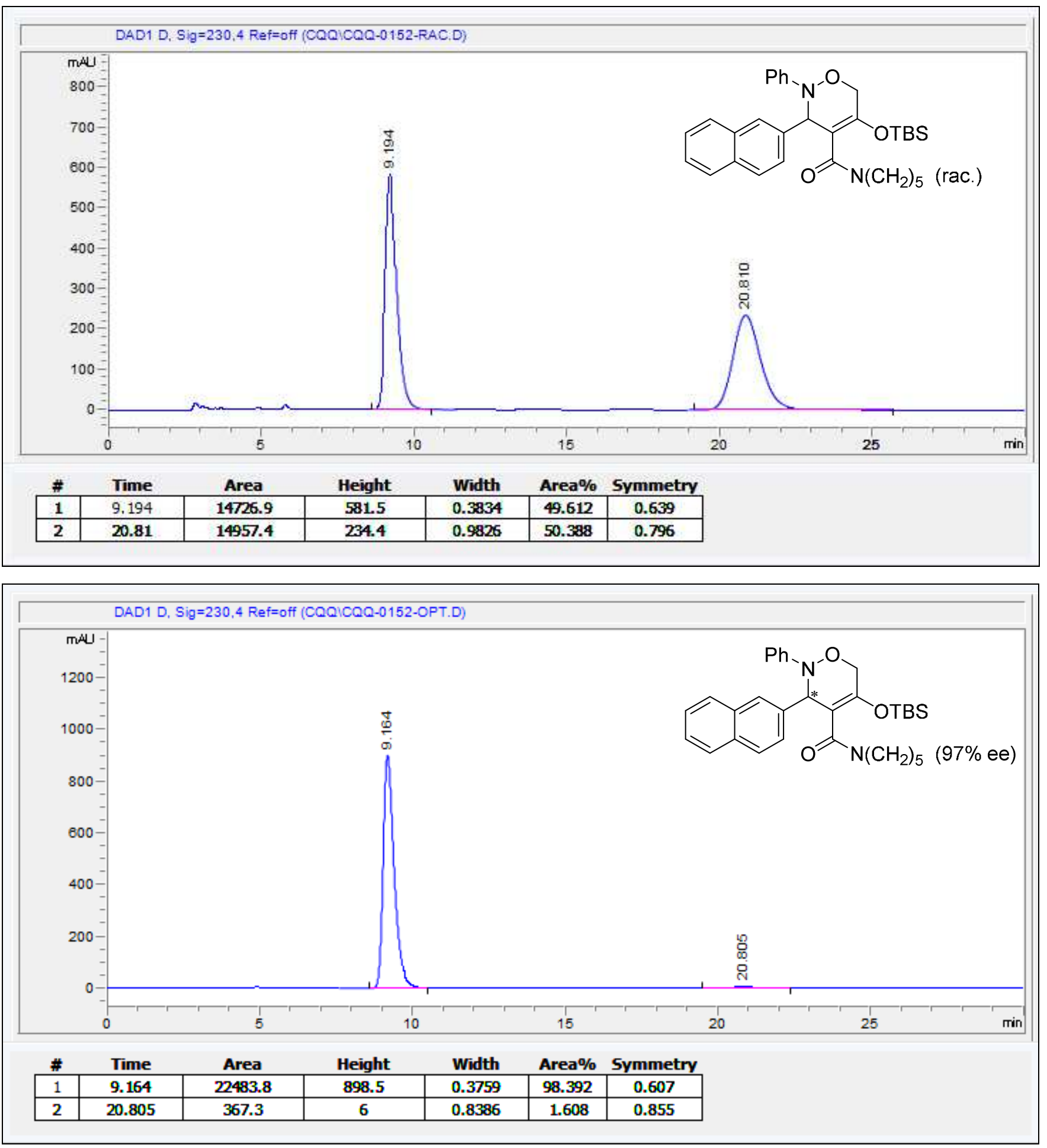
[5-(tert-Butyldimethylsilyloxy)-3-(furan-2-yl)-2-phenyl-3,6-dihydro-2H-1,2-oxazin-4-yl](piperidin1-yl)methanone (3ag)
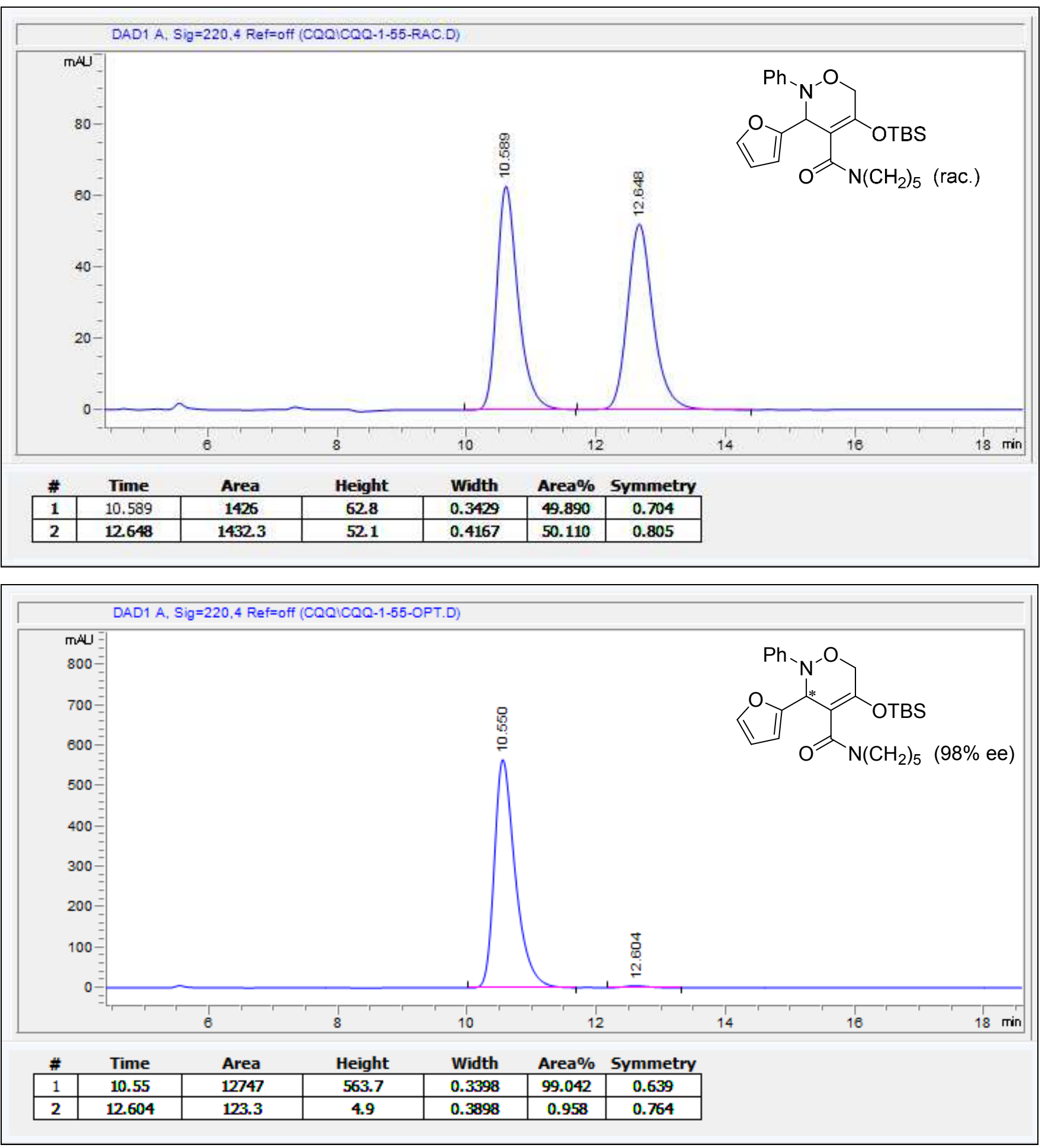
Ethyl 4-[5-(tert-Butyldimethylsilyloxy)-3-phenyl-4-(piperidine-1-carbonyl)-3,6-dihydro-2H-1,2oxazin-2-yl]benzoate (3ah)
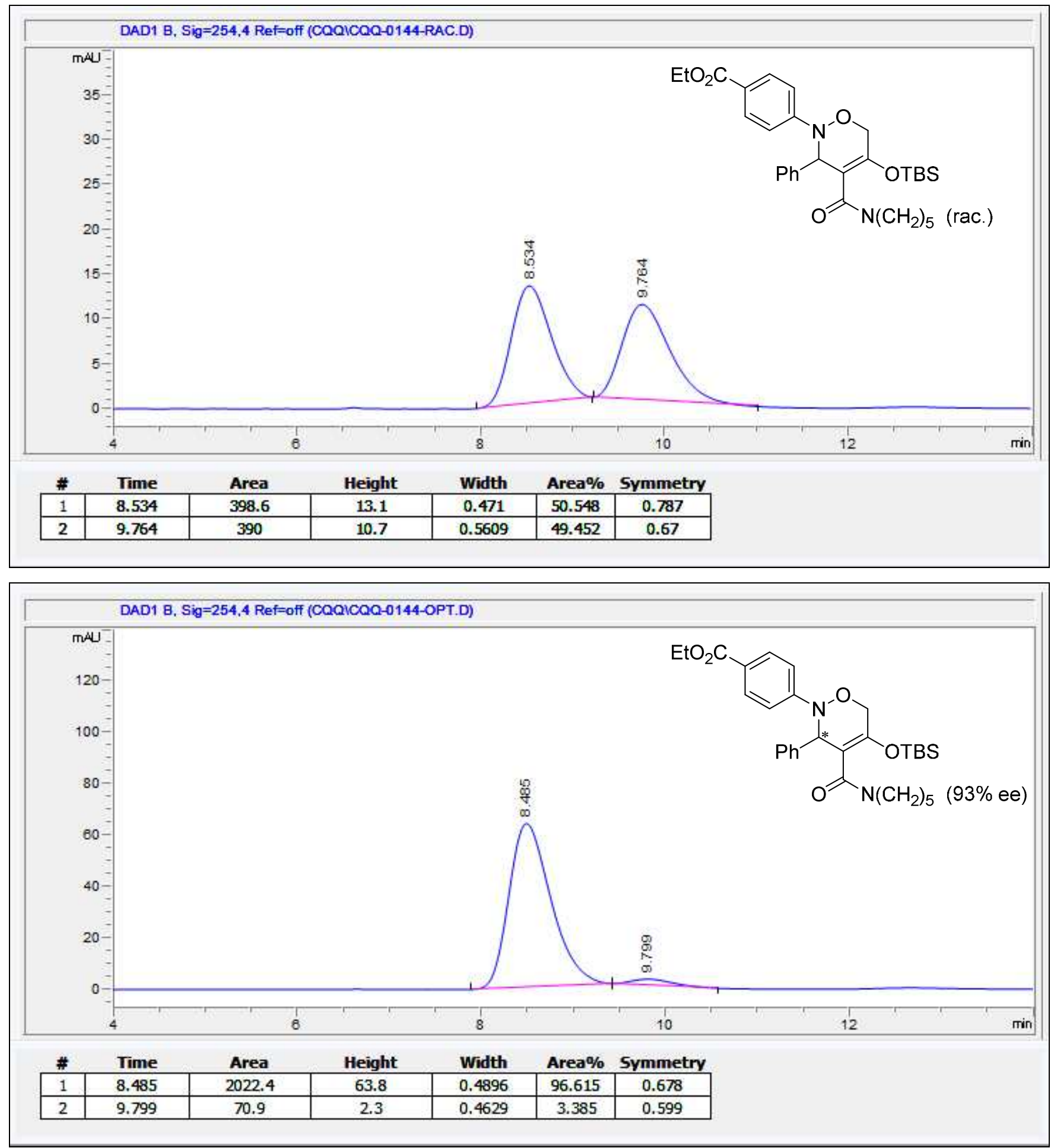
[2-(3-Bromo-4-methylphenyl)-5-(tert-butyldimethylsilyloxy)-3-phenyl-3,6-dihydro-2H-1,2-oxazin4-yl](piperidin-1-yl)methanone (3ai)
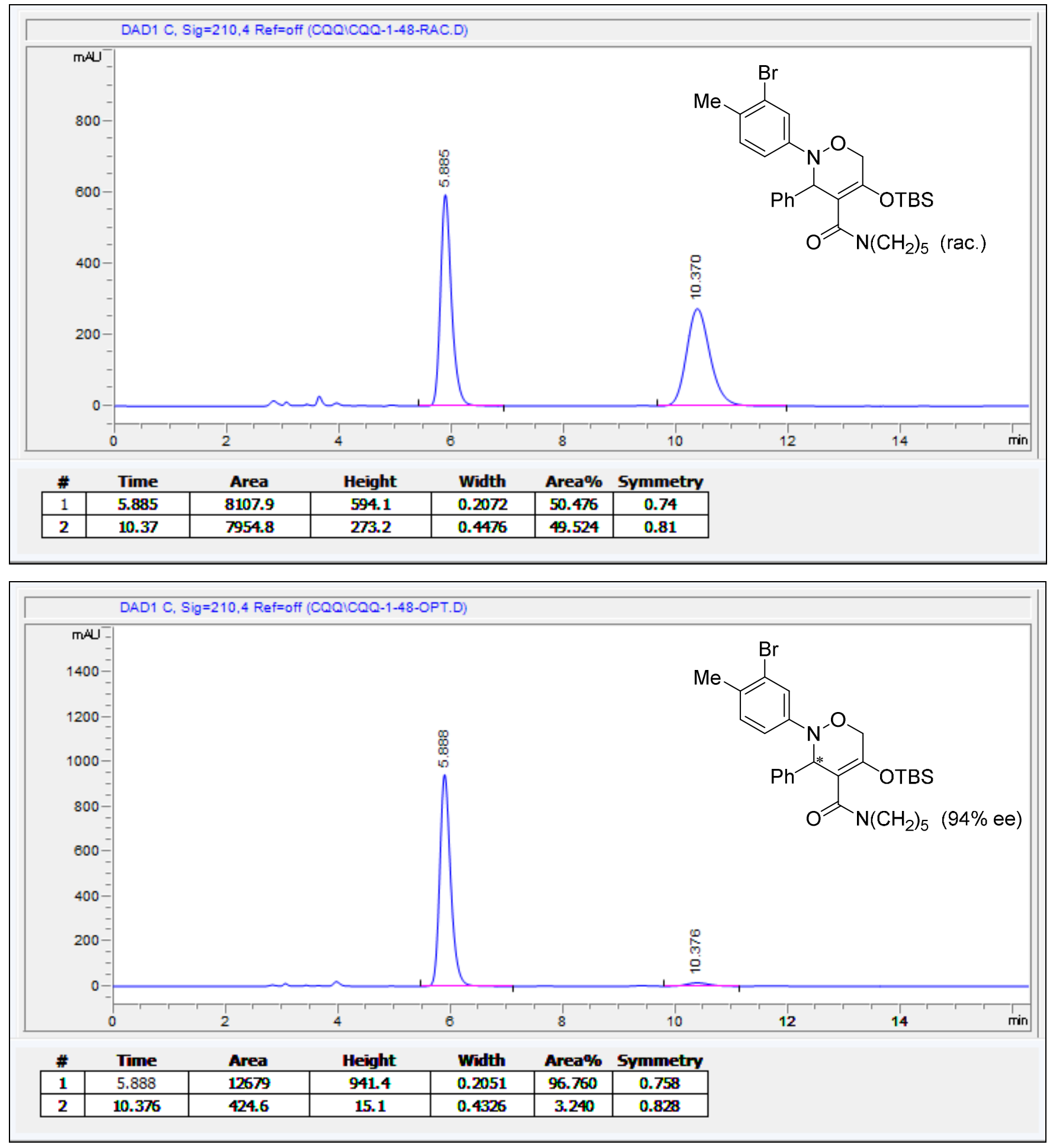
[2-Benzyl-5-(tert-butyldimethylsilyloxy)-3-cyclohexyl-3,6-dihydro-2H-1,2-oxazin-4-yl](piperidin-1yl)methanone (3aj)
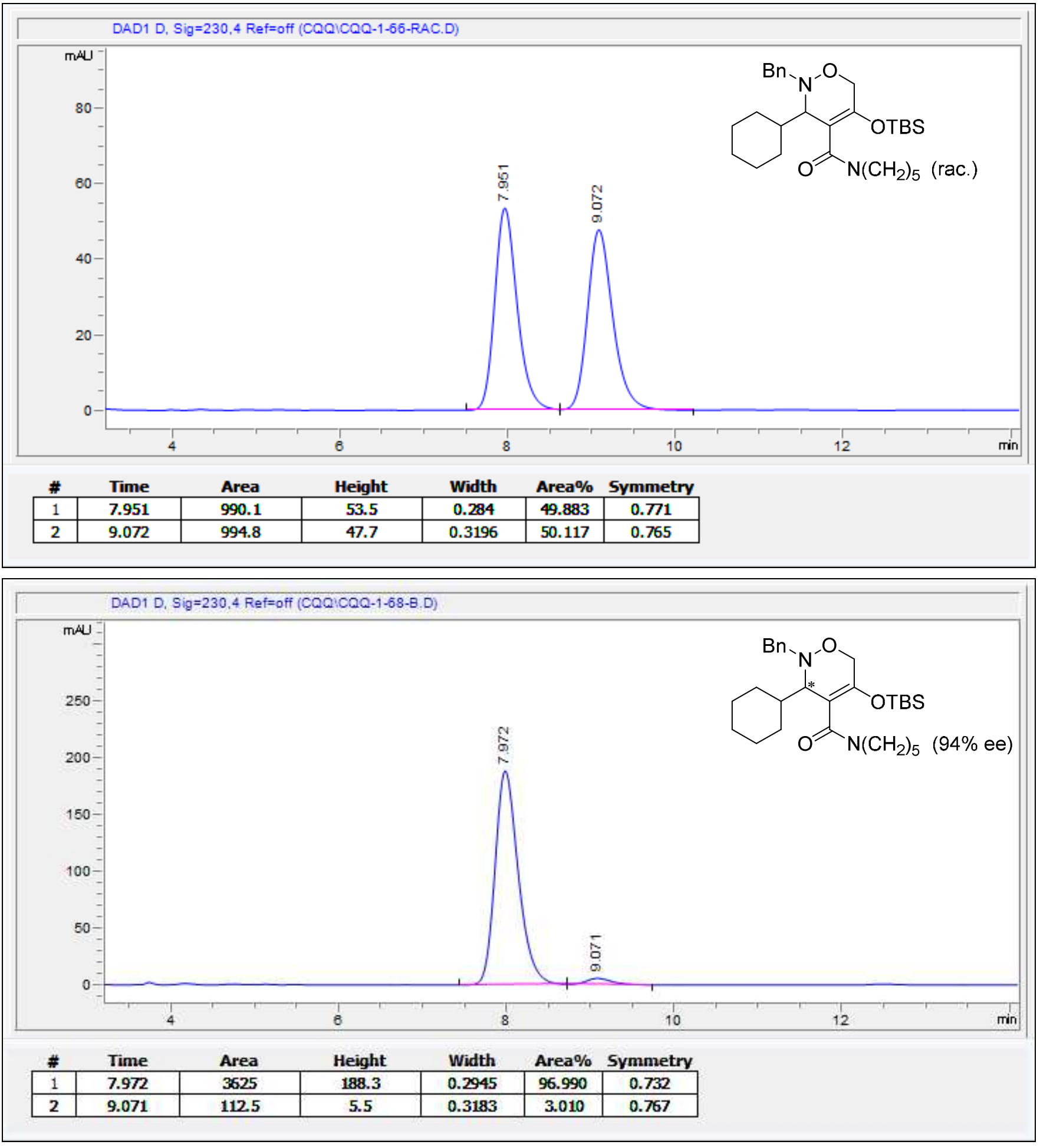
5-(tert-Butyldimethylsilyloxy)- $N, N$-dimethyl-2,3-diphenyl-3,6-dihydro-2H-1,2-oxazine-4-carboxamide (3ba)
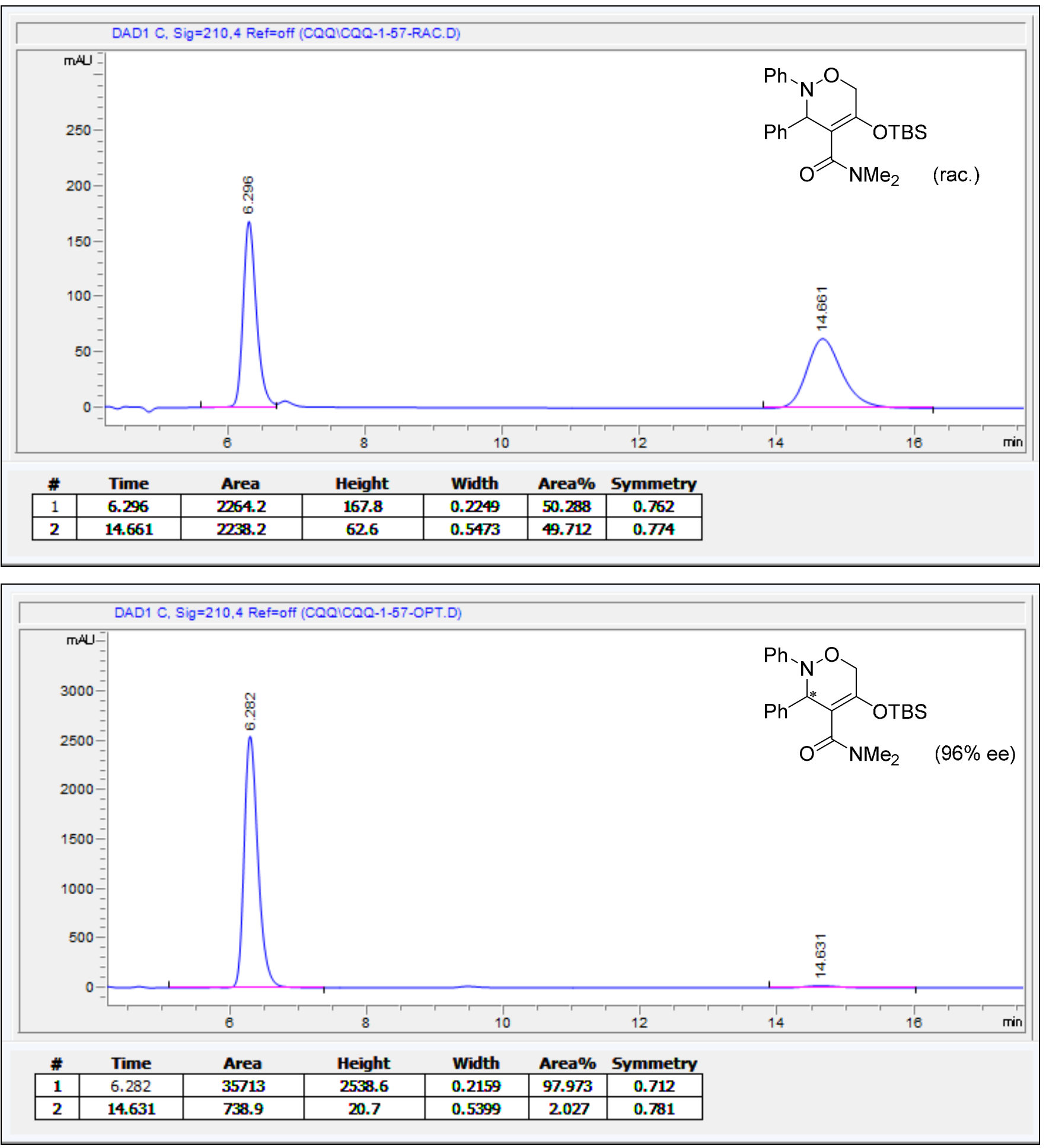
[5-(tert-Butyldimethylsilyloxy)-6-methyl-2,3-diphenyl-3,6-dihydro-2H-1,2-oxazin-4-yl](piperidin-1yl)methanone (3ca)
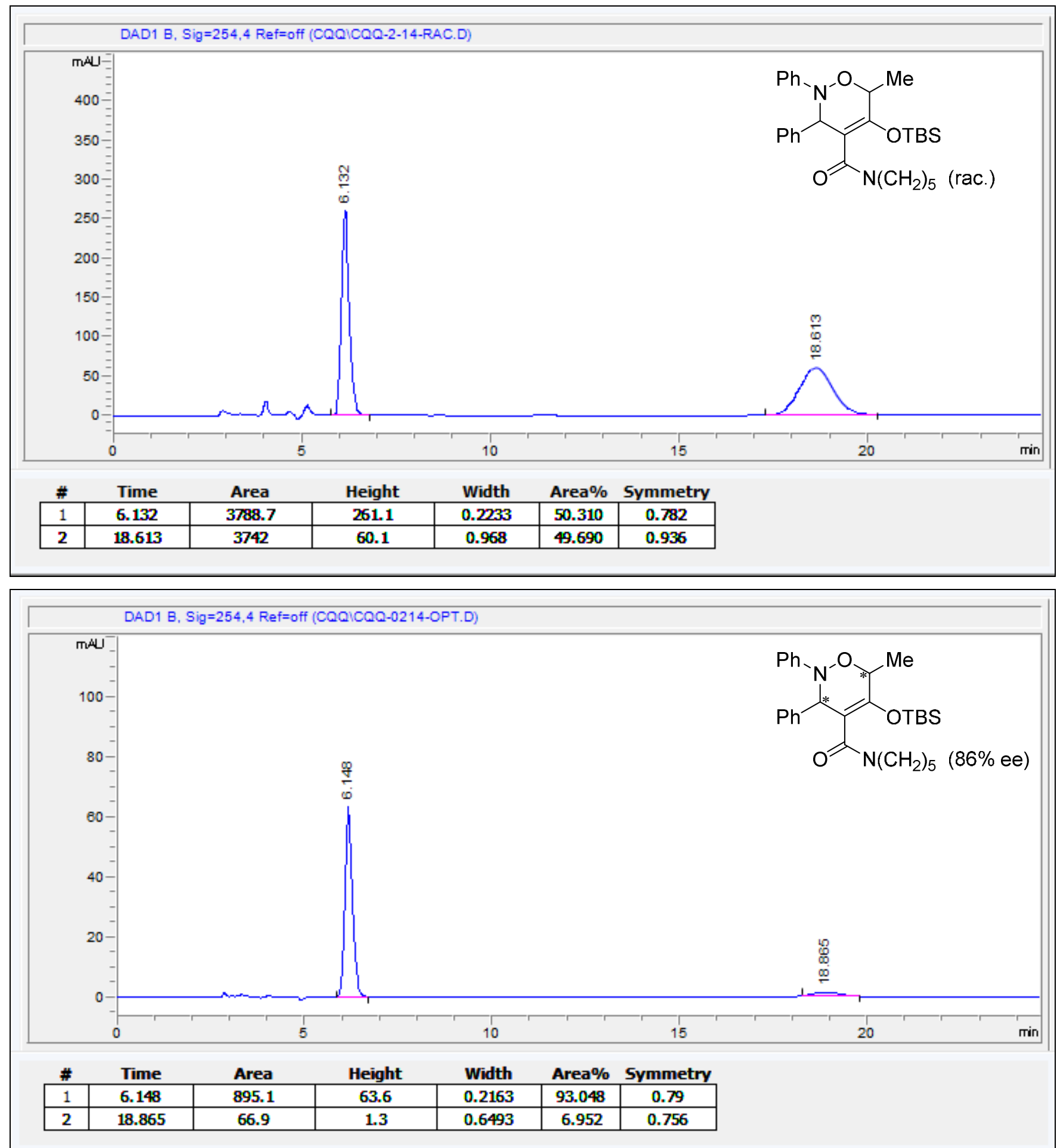
[5-(tert-Butyldimethylsilyloxy)-6-ethyl-2,3-diphenyl-3,6-dihydro-2H-1,2-oxazin-4-yl](piperidin-1yl)methanone (3da)
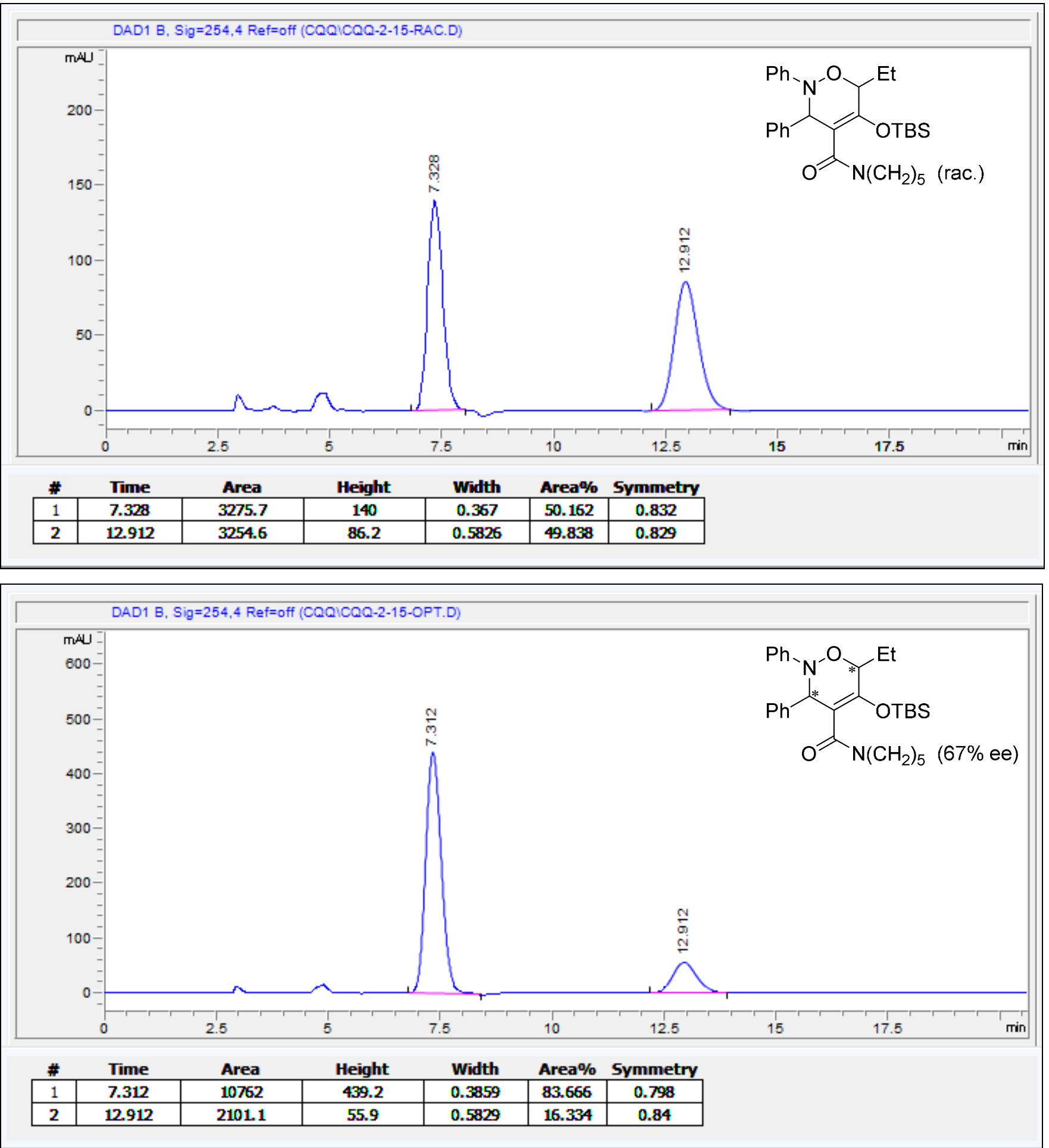